\title{
Identification of Contamination Control Strategy for Fluid Power System Using an Inexact Chance-Constrained Integer Program
}

\author{
Y. Q. Huang, S. L. Nie, and H. Ji \\ College of Mechanical Engineering and Applied Electronics Technology, Beijing University of Technology, Beijing 100124, China \\ Correspondence should be addressed to S. L. Nie; niesonglin@tom.com
}

Received 27 October 2013; Accepted 2 January 2014; Published 12 May 2014

Academic Editor: Yuefei Huang

Copyright (c) 2014 Y. Q. Huang et al. This is an open access article distributed under the Creative Commons Attribution License, which permits unrestricted use, distribution, and reproduction in any medium, provided the original work is properly cited.

\begin{abstract}
An inexact chance-constrained integer programming (ICIP) method is developed for planning contamination control of fluid power system (FPS). The ICIP is derived by incorporating chance-constrained programming (CCP) within an interval mixed integer linear programming (IMILP) framework, such that uncertainties presented in terms of probability distributions and discrete intervals can be handled. It can also help examine the reliability of satisfying (or risk of violating) system constraints under uncertainty. The developed method is applied to a case of contamination control planning for one typical FPS. Interval solutions associated with risk levels of constraint violation are obtained. They can be used for generating decision alternatives and thus help designers identify desired strategies under various environmental, economic, and system reliability constraints. Generally, willingness to take a higher risk of constraint violation will guarantee a lower system cost; a strong desire to acquire a lower risk will run into a higher system cost. Thus, the method provides not only decision variable solutions presented as stable intervals but also the associated risk levels in violating the system constraints. It can therefore support an in-depth analysis of the tradeoff between system cost and system-failure risk.
\end{abstract}

\section{Introduction}

Contamination has long been recognized as one of the major causes of components' wear, work-failure, and related downtime of fluid power system (FPS). Although a number of studies were undertaken for dealing with the contamination problems in the FPS [1-7], they focused on the contaminant sensitivity analysis for single system component, the ingression estimation of contaminant particle, and the online monitoring of cleanliness level. They had difficulties in reflecting the filtration systems from the perspective of the whole FPS as well as analyzing the economic effects of system maintenance.

Actually, in contamination control of FPS, uncertainties may exist in related costs and impact factors such as the contaminant-ingression/generation rate, component's contaminant-sensitivity, and filter's contaminant-holding capacity. These complexities may be further multiplied by not only interactions among uncertain components, but also their associations with economic penalties if major contamination accidents occur. It is thus desirable to develop effective optimization methods for reflecting the inherent complexities and uncertainties as well as effective management measures for mitigating the effect of contamination in FPS. Nie et al. developed several inexact optimization methods for addressing uncertainties and nonlinearities in the contamination control and filter management strategies of the fluid power systems [8-11]. For example, Nie et al. developed an interval-parameter integer nonlinear programming method to deal with the uncertainties in the contamination control of FPS, where the uncertainties have been presented into interval numbers [8]. Nie et al. developed an interval-fuzzy quadratic programming method for planning contamination control of the fluid power systems under uncertainty, which incorporated techniques of interval-parameter programming (IPP) and fuzzy quadratic programming within a general framework to handle uncertainties expressed in terms of interval values and fuzzy sets; multiple control variables were 
adopted to tackle independent uncertainties in the model's right-hand side, and fuzzy quadratic terms were used to minimize the variation of satisfaction degrees among the constraints [9]. Nie et al. proposed an independent variables controlled interval-fuzzy nonlinear programming method for the assessment of filter allocation and replacement strategies in FPS under uncertainty; by introducing independent control variables and L-R fuzzy numbers into the interval nonlinear programming model framework, the developed method can address the independent characteristics of constraints uncertainty [10]. Nie et al. advanced an interval-fuzzy chance-constrained integer programming (IFCIP) approach for dealing with uncertainties presented in terms of fuzzy sets, intervals, and random variables [11].

Stochastic mathematical programming (SMP) can deal with uncertainties based on the probability theory, in which probabilistic information for a limited number of uncertain parameters can be incorporated within the optimization framework. The SMP methods are effective when the lefthand-side coefficients are deterministic while the right-handside coefficients are uncertain but with known probability distributions [12]. The SMP methods include chanceconstrained programming, two-stage stochastic programming, and multistage stochastic programming (abbreviated as CCP, TSP, and MSP). CCP can effectively reflect the reliability of satisfying (or risk of violating) system constraints under uncertainty $[13,14]$. In fact, CCP does not require that all of the constraints be totally satisfied. Instead, they can be satisfied in a proportion of cases with given probabilities [15-18]. There have been many applications of CCP methods to environmental management problems [19-24]. Huang advanced two fuzzy chance-constrained programming methods for capital budgeting and investment problems that are involved in fuzzy-random parameters $[25,26]$; Li et al. proposed a multistage fuzzy chance-constrained programming approach for dealing with uncertainties expressed as fuzzy sets and probabilities in water resources management systems [17]. Although the CCP can deal with uncertainties presented as probability distributions, linear constraints in the CCP can only reflect the case when the left-hand-side coefficient is deterministic, while the set of feasible constraints becomes much more complicated or numerous if both left- and righthand sides are both random variables [21, 27-30]. However, the CCP is effective in reflecting probability distributions of the constraints' right-hand sides but not so much for independent uncertainties of the left-hand-side coefficients in each constraint or the objective function; moreover, the quality of many uncertainties is often not good enough to be presented as probability distributions [21]. These difficulties may affect practical applicability of the CCP method.

Interval-parameter programming (IPP) is an alternative for handling uncertainties presented as interval numbers in the model's left- and/or right-hand sides as well as those that cannot be quantified as membership or distribution functions [31]. IPP can be transformed into two deterministic submodels, which correspond to upper- and lower-bounds of the desired objective function value. However, an IPP model may become infeasible when its right-hand-side parameters are highly uncertain [32]. In fact, in the contamination control process of FPS, many parameters may appear uncertain and their interrelationships are complicated, such as fluid flow, filtration ratio, contaminant ingression rate, contaminant generation rate, component tolerance level, filter costs, and system maintenance fees. These uncertainties can be quantified as probability density functions (PDFs), while the others may exist as discrete intervals. For example, an engineering designer/manager may know that the contaminantgeneration-rate in a FPS fluctuates within a certain interval, but he/she may find it difficult to state its reliable probability distribution [33]. Therefore, one potential approach for better accounting for the uncertainties, as well as the relevant system reliabilities, is to incorporate the CCP into the interval mixed integer linear programming (IMILP) framework. This would then lead to an inexact chance-constrained integer programming (ICIP) method.

As an extension of the previous works, this study aims to develop such an inexact chance-constrained integer programming (ICIP) method for contamination control and filter management under uncertainty. This method will incorporate techniques of interval, integer, and chanceconstrained programming within a general framework to reflect a variety of uncertainties existing in the system parameters. It can also help examine the reliability of satisfying (or risk of violating) system constraints under uncertainty and thus quantify the cost of violating the constraints under varied risk levels. The method will then be applied to a case of contamination control planning for FPS. The results can be used for generating a range of decision alternatives under various system conditions and thus helping FPS managers to identify desired contamination control policies.

\section{Methodology}

2.1. Interval Integer Programming. According to Huang et al. [34], an interval mixed integer liner programming (IMILP) problem can be expressed as follows:

$$
\begin{array}{ll}
\text { Minimize } & f^{ \pm}=C^{ \pm} X^{ \pm} \\
\text {Subject to: } & A^{ \pm} X^{ \pm} \leq B^{ \pm} \\
& X^{ \pm} \geq 0,
\end{array}
$$

where $A^{ \pm} \in\left\{R^{ \pm}\right\}^{m \times n}, B^{ \pm} \in\left\{R^{ \pm}\right\}^{m \times 1}, C^{ \pm} \in\left\{R^{ \pm}\right\}^{1 \times n}, X^{ \pm} \in$ $\left\{R^{ \pm}\right\}^{n \times 1}, R^{ \pm}$denotes a set of interval numbers, $f^{ \pm}$refers to an interval objective function, and the decision variables $\left(X^{ \pm}\right)$ can be sorted into two categories: continuous and binary. The IMILP model can be transformed into two deterministic submodels corresponding to upper- and lower-bounds of the objective function value [34]. By solving the two submodels, interval solutions can be obtained.

2.2. Chance-Constrained Programming. In a real-world contamination control problem, randomness in other righthand-side parameters, such as component contamination sensitivities and contaminant-holding capacities, also needs to be reflected. For example, the contaminant-holding capacity may be fixed with a level of probability, which represents 
the admissible risk of violating the uncertain capacity constraint. However, the interval mathematical programming method has difficulties in reflecting uncertainties expressed as probabilistic distributions. Chance-constrained programming (CCP) method can be used for dealing with the above type of uncertainty and analyzing the risk of violating the uncertain constraints [14]. In CCP, it is required that the constraints should be satisfied under given probabilities $[15$, 19-24, 35, 36]. Consider a general probabilistic stochastic linear problem as follows:

$$
\begin{array}{ll}
\text { Min } & C(t) X \\
\text { Subject to: } & A(t) X \leq B(t) \\
& X \geq 0,
\end{array}
$$

where $X$ is a vector of decision variables and $A(t), B(t)$, and $C(t)$ are sets with random elements defined on a probability space $T, t \in T[14,30]$. The CCP approach solves the above model by converting it into a deterministic version through (i) fixing a certain level of probability $p_{i}\left(p_{i} \in[0,1]\right)$ for uncertain constraint $i$, which represents the admissible risk of violating constraint $i$, and (ii) imposing the condition that the constraint should be satisfied with at least a probability level of $1-p_{i}$. The feasible solution set is thus subject to the following constraints $[21,37]$ :

$$
\begin{aligned}
& \operatorname{Pr}\left[\left\{A_{i}(t) X \leq b_{i}(t)\right\}\right] \\
& \quad \geq 1-q_{i}, \quad A_{i}(t) \in A(t), \quad i=1,2, \ldots, m .
\end{aligned}
$$

Constraint (3) is generally nonlinear, and the set of feasible constraints is convex only for some particular distributions and certain levels of $p_{i}$, such as the cases when (i) $a_{i j}$ are deterministic and $b_{i}$ are random (for all $p_{i}$ values); (ii) $a_{i j}$ and $b_{i}$ are discrete random coefficient, with $p_{i} \geq$ $\max _{r=1,2, \ldots, R}\left(1-q_{r}\right)$, where $q_{r}$ is the probability associated with realization $r$; or (iii) $a_{i j}$ and $b_{i}$ have Gaussian distributions, with $p_{i} \geq 0.5$ [27]. When elements of $a_{i j}$ are deterministic and $b_{i}(t)$ are random, constraint (3) can be converted into a linear one as follows:

$$
A_{i}(t) X \leq b_{i}(t)^{p_{i}}, \quad \forall i
$$

where $b_{i}(t)^{p i}=F_{i}^{-1}\left(p_{i}\right)$, given the cumulative distribution function $(\mathrm{CDF})$ of $b_{i}$ (i.e., $\left.F_{i}\left(b_{i}\right)\right)$ and the probability of violating constraint $i$ (i.e., $p_{i}$ ). The problem with constraint (4) is that linear constraints can only reflect the case when the left-hand-side coefficients $(A)$ are deterministic. If both left- and right-hand sides $(A$ and $B)$ are uncertain, the set of feasible constraints may become more complicated [21, 27-30]. To reflect randomness of the objective function in IIP model, an "equivalent" deterministic objective is usually defined in the CCP approach. There are four main options: (i) optimization of mean value, (ii) minimization of variance or other dispersion parameters, (iii) minimization of risks, and (iv) maximization of the fractile (or Kataoka's problem). However, these considerations may be unable to effectively handle independent uncertainties in $c_{j}$ and communicate them into the constraints.
One potential approach for better accounting for multiple uncertainties that exist in both left- and right-hand sides (of the constraints) as well as objective-function coefficients (i.e., $A, B$, and $C$ ) is to incorporate the CCP within the above IMILP framework, where intervals and probability distributions could be reflected. This leads to an interval chance-constrained integer programming (ICIP) model as follows:

$$
\begin{array}{ll}
\text { Maximize } & \lambda^{ \pm} \\
\text {Subject to: } & C^{ \pm} X^{ \pm} \leq f^{+}-\lambda^{ \pm}\left(f^{+}-f^{-}\right) \\
& A_{i}^{ \pm}(t) X^{ \pm} \leq b_{i}(t)^{q_{i}^{ \pm}} \quad \forall i, \quad i=1,2, \ldots, m ; \\
& A_{i}^{ \pm}(t) \in A^{ \pm}(t) \\
& x^{ \pm} \geq 0, \quad x_{j}^{ \pm} \in X^{ \pm} ; j=1,2, \ldots, n_{1} \\
& 0 \leq \lambda^{ \pm} \leq 1,
\end{array}
$$

where $\lambda^{ \pm}$is a control variable, representing the degree of satisfaction for fuzzy decision. Figure 1 shows the framework of the ICIP. It is indicated that the ICIP integrates techniques of interval sets, integer and chance-constrained within a general framework. Each technique has its unique contribution in enhancing the model's capacities for tackling uncertainties and dynamics. The ICIP can thus deal with uncertainties described as discrete intervals sets and their combinations. Based on an interactive algorithm, interval solutions associated with levels of system-failure risk can be obtained through solving two submodels sequentially. The solutions are useful in generating desired decision alternatives with the relationships among system cost, satisfaction degree, and constraint-violation risk being quantified.

\section{Case Study}

3.1. Statement of Problem. Since the fluid power system can inevitably be contaminated by large amounts of contaminants, filters have to be adopted to mitigate the contamination level and thus guarantee the critical hydraulic components not being polluted and/or destroyed. The major functions of filters are to remove contaminant particles and thus keep the system contamination at a safe level (i.e., lower than the tolerance levels of various system components). Assume that four types of filters (suction, pressure, return, and bypass filters) would be installed in the study system. The detailed nomenclatures for the variables and parameters are provided in the appendix. The binary variables (i.e., decision variables, $F_{k}$ and $A_{k n}$ ) can be employed to identify (i) whether or not particular filter needs to be installed and (ii) whether or not the existing filter needs to be replaced. For example, if filter $k$ exists then $F_{k}=1$; otherwise $F_{k}=0$; similarly, if filter $k$ needs to be replaced in period, then $A_{k n}=1$; otherwise $A_{k n}=0$.

Additionally, the filter fineness plays a crucial role in ensuring the reliability and service life of pump in the FPS. The management of filters is of importance during the operation of FPS. Installing new filters and/or replacing the existing filters frequently may lead to an increased operation 


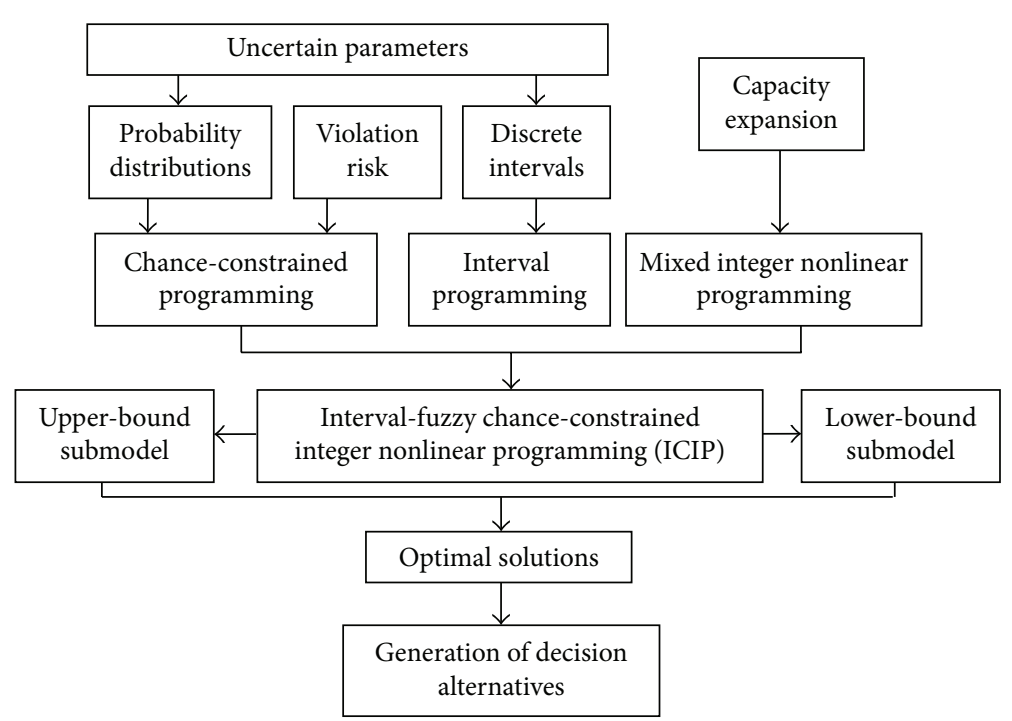

Figure 1: Framework of the ICIP model.

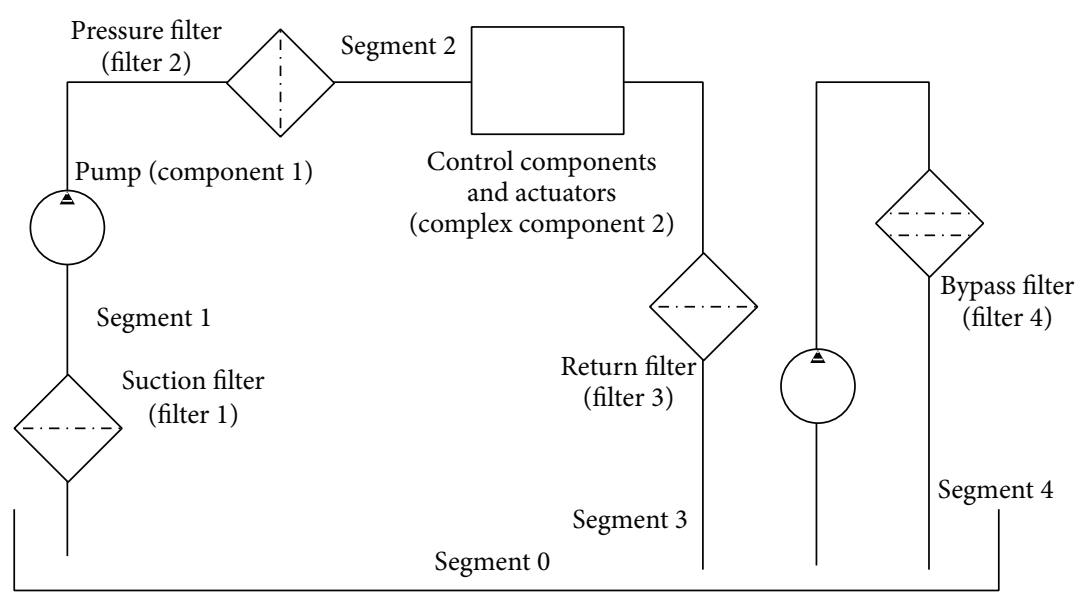

Oil reservoir (component 3)

Figure 2: Typical hydraulic system with bypass filtration system.

cost. However, misact in installing filters and/or prolonging the replacement period of the filters may pose serious contamination threats on the FPS. Several factors such as operation cost, replacement period, system performance, service life of pump, and fineness of filters may be complex and conflicted with each other. Therefore, it is desirable to select filter fineness and plan the replacement period of filters properly and to identify how to choose suitable filters (including filter housing and filter element) and/or when to replace the existing filters at an appropriate time which would make a tradeoff between operation cost and system-failure risk.

Consider a problem wherein a decision maker is responsible for the selection, installation, and replacement of filters in a FPS with a bypass filtration circuit. Figure 2 presents such a FPS, which includes an oil reservoir, a hydraulic pump, a suction filter, a pressure filter, a return filter, a bypass filter and several control components, and an actuator. To simplify the formal deduction of model these control components and actuator are regarded to be a complex component here.

Several assumptions are made when formulating the model, which includes the following. (1) Contaminants are evenly distributed in each segment of the fluid power system and the contamination level in any segment will not vary within a working period of filter; (2) filtration ratio of the filter is constant at any time for a given particle parameter range; (3) the other components after those filters cannot remove the contaminant particles; (4) the flow rate is assumed to be constant and the effect of system pressure and flow on the filtration efficiency is negligible; (5) all of the contaminants are considered spherical when calculating their granular mass; (6) the settling effect of the contaminants in the oil reservoir is negligible; (7) the effect of cavitation on the performance of hydraulic components due to the flow resistance of the filters is negligible; (8) the replacement of oil is out of consideration in this study. 
3.2. Model Formulation. In such a typical hydraulic system (as shown in Figure 2), contaminant mainly comes from both outside environment and inside hydraulic components. Since the contamination ingression rate (CIR) plays an important part in removing contaminant, a further research should be conducted concerning more details about CIR. It is known that CIR is not a constant during the operation of the system. At the threshold of the system running, the value of CIR is at the top and starts to decrease with an increased speed because of intense friction. Gradually, the friction surfaces become smoother and thus the reducing rate of the speed slows down and finally ends up with zero, which means that the CIR keeps being a constant.

In the CCP, the required level of probability represents the admissible risk of violating the constraints [14, 21]. Thus, the CCP can be incorporated into the concept of IIP into a general framework to deal with uncertainties in the contaminant-holding capacity of filters and component contaminant sensitivity. Based on the preceding research [8], a chance-constrained programming (CCP) model (A) can be formulated as follows:

$$
\begin{aligned}
\text { Minimize } \quad F_{\text {cost }}= & \sum_{k=1}^{4} B_{k} F_{k} \\
& +\sum_{k=1}^{4} \sum_{n=1}^{N}\left(\mathrm{ER}_{k}+\mathrm{WM}_{k}+\mathrm{LM}_{k}\right) A_{k n}
\end{aligned}
$$

subject to

$$
\begin{gathered}
\operatorname{Pr}\left\{N F_{1 n m} \leq S_{1 n m}\right\} \geq 1-p_{i}, \quad \forall n, m, \\
\operatorname{Pr}\left\{N F_{2 n m} \leq S_{2 n m}\right\} \geq 1-p_{i}, \quad \forall n, m, \\
\operatorname{Pr}\left\{\sum_{n=1}^{t} \sum_{m=1}^{M} F_{1} \times N F_{0 n m} \times\left(1-\frac{1}{\beta_{1 m}}\right) Q T\right. \\
\left.\times\left[\frac{4 \rho \pi}{3} \times\left(\frac{D_{m}}{2}\right)^{3}\right] \leq \sum_{n=1}^{t} A_{1 n} C_{1} \times 10^{15}\right\} \\
\geq 1-p_{i}, \quad \forall t=1,2, \ldots, N, \\
\operatorname{Pr}\left\{\sum_{n=1}^{t} \sum_{m=1}^{M} F_{2}\left(N F_{1 n m}+\frac{R_{1(n-1) m}}{Q}\right)\right. \\
\times\left(1-\frac{1}{\beta_{2 m}}\right) Q T\left[\frac{4 \rho \pi}{3} \times\left(\frac{D_{m}}{2}\right)^{3}\right] \\
\left.\leq \sum_{n=1}^{t} A_{2 n} C_{2} \times 10^{15}\right\} \\
\geq 1-p_{i}, \quad \forall t=1,2, \ldots, N,
\end{gathered}
$$

$$
A_{i} X \leq b_{i}^{p_{i}}, \quad \forall i,
$$

where $b_{i}^{p_{i}}=F_{i}^{-1}\left(p_{i}\right)$, given the cumulative distribution function $(\mathrm{CDF})$ of $b_{i}$ (i.e., $\left.F_{i}\left(b_{i}\right)\right)$ and the probability of 
violating constraint $i$ (i.e., $p_{i}$ ). Consequently, the above CCP model can be converted into a linear model (B) as follows:

$$
\begin{aligned}
\text { Minimize } F_{\text {cost }}= & \sum_{k=1}^{4} B_{k} F_{k} \\
& +\sum_{k=1}^{4} \sum_{n=1}^{N}\left(\mathrm{ER}_{k}+\mathrm{WM}_{k}+\mathrm{LM}_{k}\right) A_{k n}
\end{aligned}
$$

subject to

$$
\begin{aligned}
& N F_{1 n m} \leq S_{1 n m}^{(p i)}, \quad \forall n, m, \\
& N F_{2 n m} \leq S_{2 n m}^{(p i)}, \quad \forall n, m, \\
& \sum_{n=1}^{t} \sum_{m=1}^{M} F_{1} \times N F_{0 n m}\left(1-\frac{1}{\beta_{1 m}}\right) Q T \\
& \times\left[\frac{4 \rho \pi}{3} \times\left(\frac{D_{m}}{2}\right)^{3}\right] \\
& \leq \sum_{n=1}^{t} A_{1 n} C_{1}^{(p i)} \times 10^{15}, \quad \forall t=1,2, \ldots, N, \\
& \sum_{n=1}^{t} \sum_{m=1}^{M} F_{2}\left(N F_{1 n m}+\frac{R_{1(n-1) m}}{Q}\right)\left(1-\frac{1}{\beta_{2 m}}\right) Q T \\
& \times\left[\frac{4 \rho \pi}{3} \times\left(\frac{D_{m}}{2}\right)^{3}\right] \\
& \leq \sum_{n=1}^{t} A_{2 n} C_{2}^{(p i)} \times 10^{15}, \quad \forall t=1,2, \ldots, N \\
& \sum_{n=1}^{t} \sum_{m=1}^{M} F_{3}\left(N F_{2 n m}+\frac{R_{2(n-1) m}}{Q}\right)\left(1-\frac{1}{\beta_{3 m}}\right) Q T \\
& \times\left[\frac{4 \rho \pi}{3} \times\left(\frac{D_{m}}{2}\right)^{3}\right] \\
& \leq \sum_{n=1}^{t} A_{3 n} C_{3}^{(p i)} \times 10^{15}, \quad \forall t=1,2, \ldots, N, \\
& \sum_{n=1}^{t} \sum_{m=1}^{M} F_{4}\left(N F_{3 n m}+\frac{R_{3(n-1) m}}{Q}\right)\left(1-\frac{1}{\beta_{4 m}}\right) Q T \\
& \times\left[\frac{4 \rho \pi}{3} \times\left(\frac{D_{m}}{2}\right)^{3}\right] \\
& \leq \sum_{n=1}^{t} A_{4 n} C_{4}^{(p i)} \times 10^{15} \quad \forall t=1,2, \ldots, N,
\end{aligned}
$$

$$
\begin{aligned}
& N F_{0(n+1) m}=\left(N F_{3 n m}-N F_{0 n m}\right) \frac{Q T}{V}+\frac{R_{3 n m} T}{V} \\
& +N F_{0 n m}+\left(N F_{4 n m}-N F_{3 n m}\right) \frac{Q_{b} T}{V}, \\
& F_{k}= \begin{cases}1 & \text { if filter needs to be installed } \\
0 & \text { otherwise }\end{cases} \\
& \forall k \text {, } \\
& A_{k n}= \begin{cases}1 & \text { if filter needs to be replaced } \\
0 & \text { otherwise }\end{cases} \\
& \forall k, n \text {, } \\
& A_{k n} \leq F_{k}, \quad \forall k, n .
\end{aligned}
$$

The above CCP model can handle all right-hand-side uncertainties expressed as probability distributions. However, the linear constraints only correspond to cases when the left-hand-side coefficients are deterministic. Although the CCP approach can deal with left-hand-side uncertainties presented as probability density functions, three limitations exist: (1) the resulting nonlinear model would be associated with a number of difficulties in global-optimum acquisition; (2) it is unable to handle independent uncertainties in objective coefficients [38]; (3) for many practical problems, the quality of information that can be obtained for these uncertainties is mostly not good enough to be presented as probability distributions. Thus, for uncertainties in left-handside parameters (e.g., contamination level and component contamination sensitivity and contaminant retaining capacity), an extended consideration would be the introduction of interval parameters into the model (C). This leads to an inexact chance-constrained integer programming (ICIP) model as follows:

$$
\begin{aligned}
\text { Minimize } F_{\text {cost }}^{ \pm}= & \sum_{k=1}^{4} B_{k}^{ \pm} F_{k}^{ \pm} \\
& +\sum_{k=1}^{4} \sum_{n=1}^{N}\left(\mathrm{ER}_{k}^{ \pm}+\mathrm{WM}_{k}^{ \pm}+\mathrm{LM}_{k}^{ \pm}\right) A_{k n}^{ \pm}
\end{aligned}
$$

subject to

$$
\begin{gathered}
N F_{1 n m}^{ \pm} \leq S_{1 n m}^{(p i) \pm}, \quad \forall n, m, \\
N F_{2 n m}^{ \pm} \leq S_{2 n m}^{(p i) \pm}, \quad \forall n, m, \\
\sum_{n=1}^{t} \sum_{m=1}^{M} F_{1} \times N F_{0 n m}^{ \pm}\left(1-\frac{1}{\beta_{1 m}}\right) Q T \\
\times\left[\frac{4 \rho \pi}{3} \times\left(\frac{D_{m}}{2}\right)^{3}\right] \\
\leq \sum_{n=1}^{t} A_{1 n}^{ \pm} C_{1}^{(p i) \pm} \times 10^{15}, \quad \forall t=1,2, \ldots, N,
\end{gathered}
$$




$$
\begin{gathered}
\sum_{n=1}^{t} \sum_{m=1}^{M} F_{2}\left(N F_{1 n m}^{ \pm}+\frac{R_{1(n-1) m}^{ \pm}}{Q}\right)\left(1-\frac{1}{\beta_{2 m}}\right) Q T \\
\times\left[\frac{4 \rho \pi}{3} \times\left(\frac{D_{m}}{2}\right)^{3}\right] \\
\leq \sum_{n=1}^{t} A_{2 n}^{ \pm} C_{2}^{(p i) \pm} \times 10^{15}, \quad \forall t=1,2, \ldots, N \\
\sum_{n=1}^{t} \sum_{m=1}^{M} F_{3}\left(N F_{2 n m}^{ \pm}+\frac{R_{2(n-1) m}^{ \pm}}{Q}\right)\left(1-\frac{1}{\beta_{3 m}}\right) Q T \\
\times\left[\frac{4 \rho \pi}{3} \times\left(\frac{D_{m}}{2}\right)^{3}\right]
\end{gathered}
$$$$
\leq \sum_{n=1}^{t} A_{3 n}^{ \pm} C_{3}^{(p i) \pm} \times 10^{15}, \quad \forall t=1,2, \ldots, N
$$$$
\sum_{n=1}^{t} \sum_{m=1}^{M} F_{4}\left(N F_{3 n m}^{ \pm}+\frac{R_{3(n-1) m}^{ \pm}}{Q}\right)\left(1-\frac{1}{\beta_{4 m}}\right) Q T
$$$$
\times\left[\frac{4 \rho \pi}{3} \times\left(\frac{D_{m}}{2}\right)^{3}\right]
$$$$
\leq \sum_{n=1}^{t} A_{4 n}^{ \pm} C_{4}^{(p i) \pm} \times 10^{15}, \quad \forall t=1,2, \ldots, N,
$$$$
N F_{0(n+1) m}^{ \pm}=\left(N F_{3 n m}^{ \pm}-N F_{0 n m}^{ \pm}\right) \frac{Q T}{V}+\frac{R_{3 n m}^{ \pm} T}{V}
$$$$
+N F_{0 n m}^{ \pm}+\left(N F_{4 n m}^{ \pm}-N F_{3 n m}^{ \pm}\right) \frac{Q_{b} T}{V}
$$

$$
F_{k}= \begin{cases}1 & \text { if filter needs to be installed } \\ 0 & \text { otherwise }\end{cases}
$$

$\forall k$,

$A_{k n}^{ \pm}= \begin{cases}1 & \text { if filter needs to be replaced } \\ 0 & \text { otherwise }\end{cases}$

$$
\begin{aligned}
& \forall k, n, \\
& A_{k n}^{ \pm} \leq F_{k}, \quad \forall k, n,
\end{aligned}
$$

where $F_{\text {cost }}^{ \pm}, B_{k}^{ \pm}, \mathrm{ER}_{k}^{ \pm}, \mathrm{WM}_{k}^{ \pm}, \mathrm{LM}_{k}^{ \pm}, A_{k n}^{ \pm}, C_{i}^{(q i) \pm}, S_{i n m}^{(q i) \pm}, N F_{i n m}^{ \pm}$, and $R_{i n m}^{ \pm}$are interval parameters and variables; the "-" and "+" superscripts represent lower- and upper-bounds of the parameters, respectively. This ICIP model can be transformed into two deterministic submodels that correspond to the

lower- and upper-bounds of the desired objective. Interval solutions, which are feasible and stable in the given decision space, can then be obtained by solving the two submodels sequentially [34]. According to Huang [21], the submodel corresponding to the lower-bound objective $\left(F_{\text {cost }}^{-}\right)$can be firstly formulated as follows:

$$
\begin{aligned}
\text { Minimize } F_{\text {cost }}^{-}= & \sum_{k=1}^{4} B_{k}^{-} F_{k}^{-} \\
& +\sum_{k=1}^{4} \sum_{n=1}^{N}\left(\mathrm{ER}_{k}^{-}+\mathrm{WM}_{k}^{-}+\mathrm{LM}_{k}^{-}\right) A_{k n}^{-}
\end{aligned}
$$

subject to

$$
\begin{gathered}
N F_{1 n m}^{-} \leq S_{1 n m}^{(p i)-}, \quad \forall n, m, \\
N F_{2 n m}^{-} \leq S_{2 n m}^{(p i)-}, \quad \forall n, m, \\
\sum_{n=1}^{t} \sum_{m=1}^{M} F_{1} \times N F_{0 n m}^{-}\left(1-\frac{1}{\beta_{1 m}}\right) Q T \\
\times\left[\frac{4 \rho \pi}{3} \times\left(\frac{D_{m}}{2}\right)^{3}\right] \\
\leq \sum_{n=1}^{t} A_{1 n}^{-} C_{1}^{(p i)-} \times 10^{15}, \quad \forall t=1,2, \ldots, N, \\
\sum_{n=1}^{t} \sum_{m=1}^{M} F_{2}\left(N F_{1 n m}^{-}+\frac{R_{1(n-1) m}^{-}}{Q}\right)\left(1-\frac{1}{\beta_{2 m}}\right) Q T \\
\times\left[\frac{4 \rho \pi}{3} \times\left(\frac{D_{m}}{2}\right)^{3}\right] \\
\leq \sum_{n=1}^{t} A_{4 n}^{-} C_{4}^{(p i)-} \times 10^{15}, \quad \forall t=1,2, \ldots, N, \quad \forall t=1,2, \ldots, N, \\
\leq \sum_{n=1}^{t} A_{2 n}^{-} C_{2}^{(p i)-} \times 10^{15}, \quad \forall t=1,2, \ldots, N, \\
\sum_{n=1}^{t} \sum_{m=1}^{M} F_{4 n}\left(C _ { 3 } ^ { M } \left(N F_{3 n m}^{-}+\frac{R_{3(n-1) m}^{-}}{Q} \times 10^{15},\left(1-\frac{1}{\beta_{4 m}}\right) Q T\right.\right. \\
\times \sum_{n=1}\left(N F_{2 n m}^{-}+\frac{R_{2(n-1) m}^{-}}{Q}\right)\left(1-\frac{1}{\beta_{3 m}}\right) Q T \\
\times\left[\frac{4 \rho \pi}{3} \times\left(\frac{D_{m}}{2}\right)^{3}\right]
\end{gathered}
$$




$$
\begin{aligned}
N F_{0(n+1) m}^{-}= & \left(N F_{3 n m}^{-}-N F_{0 n m}^{-}\right) \frac{Q T}{V}+\frac{R_{3 n m}^{-} T}{V} \\
& +N F_{0 n m}^{-}+\left(N F_{4 n m}^{-}-N F_{3 n m}^{-}\right) \frac{Q_{b} T}{V}
\end{aligned}
$$

$F_{k}= \begin{cases}1 & \text { if filter needs to be installed } \\ 0 & \text { otherwise }\end{cases}$

$$
\forall k \text {, }
$$

$A_{k n}^{-}= \begin{cases}1 & \text { if filter needs to be replaced } \\ 0 & \text { otherwise, }\end{cases}$

$$
\begin{aligned}
& \forall k, n, \\
& A_{k n}^{-} \leq F_{k}, \quad \forall k, n .
\end{aligned}
$$

Correspondingly, the submodel corresponding to the upper-bound objective $\left(F_{\text {cost }}^{+}\right)$can be formulated as follows:

$$
\begin{aligned}
\text { Minimize } F_{\text {cost }}^{+}= & \sum_{k=1}^{4} B_{k}^{+} F_{k}^{+} \\
& +\sum_{k=1}^{4} \sum_{n=1}^{N}\left(\mathrm{ER}_{k}^{+}+\mathrm{WM}_{k}^{+}+\mathrm{LM}_{k}^{+}\right) A_{k n}^{+}
\end{aligned}
$$

subject to

$$
\begin{gathered}
N F_{1 n m}^{+} \leq S_{1 n m}^{(p i)+}, \quad \forall n, m, \\
N F_{2 n m}^{+} \leq S_{2 n m}^{(p i)+}, \quad \forall n, m, \\
\sum_{n=1}^{t} \sum_{m=1}^{M} F_{1} \times N F_{0 n m}^{+}\left(1-\frac{1}{\beta_{1 m}}\right) Q T \\
\times\left[\frac{4 \rho \pi}{3} \times\left(\frac{D_{m}}{2}\right)^{3}\right] \\
\leq \sum_{n=1}^{t} A_{1 n}^{+} C_{1}^{(p i)+} \times 10^{15}, \quad \forall t=1,2, \ldots, N, \\
\sum_{n=1}^{t} \sum_{m=1}^{M} F_{2}\left(N F_{1 n m}^{+}+\frac{R_{1(n-1) m}^{+}}{Q}\right)\left(1-\frac{1}{\beta_{2 m}}\right) Q T \\
\leq \sum_{n=1}^{t} A_{2 n}^{+} C_{2}^{(p i)+} \times 10^{15}, \quad \forall t=1,2, \ldots, N \\
\times\left[\frac{4 \rho \pi}{3} \times\left(\frac{D_{m}}{2}\right)^{3}\right]
\end{gathered}
$$

$$
\begin{aligned}
& \sum_{n=1}^{t} \sum_{m=1}^{M} F_{3}\left(N F_{2 n m}^{+}+\frac{R_{2(n-1) m}^{+}}{Q}\right)\left(1-\frac{1}{\beta_{3 m}}\right) Q T \\
& \times\left[\frac{4 \rho \pi}{3} \times\left(\frac{D_{m}}{2}\right)^{3}\right] \\
& \leq \sum_{n=1}^{t} A_{3 n}^{+} C_{3}^{(p i)+} \times 10^{15}, \quad \forall t=1,2, \ldots, N, \\
& \sum_{n=1}^{t} \sum_{m=1}^{M} F_{4}\left(N F_{3 n m}^{+}+\frac{R_{3(n-1) m}^{+}}{Q}\right)\left(1-\frac{1}{\beta_{4 m}}\right) Q T \\
& \times\left[\frac{4 \rho \pi}{3} \times\left(\frac{D_{m}}{2}\right)^{3}\right] \\
& \leq \sum_{n=1}^{t} A_{4 n}^{+} C_{4}^{(p i)+} \times 10^{15}, \quad \forall t=1,2, \ldots, N, \\
& N F_{0(n+1) m}^{+}=\left(N F_{3 n m}^{+}-N F_{0 n m}^{+}\right) \frac{Q T}{V}+\frac{R_{3 n m}^{+} T}{V} \\
& +N F_{0 n m}^{+}+\left(N F_{4 n m}^{+}-N F_{3 n m}^{+}\right) \frac{Q_{b} T}{V}, \\
& F_{k}= \begin{cases}1 & \text { if filter needs to be installed } \\
0 & \text { otherwise }\end{cases} \\
& \forall k \text {, } \\
& A_{k n}^{+}= \begin{cases}1 & \text { if filter needs to be replaced } \\
0 & \text { otherwise }\end{cases}
\end{aligned}
$$

$\forall k, n$,

$$
A_{k n}^{+} \leq F_{k}, \quad \forall k, n
$$

The above solutions provide stable intervals for the objective function value and the decision variables under different levels of risk in violating the constraints. They can be easily interpreted for generating multiple decision alternatives. The detailed solution process can be summarized as follows.

Step 1. Acquire distribution information for the system constraints (e.g., contaminant retaining capacity and contamination sensitivity of filters).

Step 2. Formulate ICIP model.

Step 3. Transform ICIP model into two submodels, where the submodel corresponding to $F_{\text {cost }}^{-}$should be firstly solved (to obtain the most optimistic decision option within the decision space) since the objective is to minimize $F_{\text {cost }}^{ \pm}$.

Step 4. Formulate $F_{\text {cost }}^{-}$submodel, including the objective function and the relevant constraints.

Step 5. Solve the $F_{\text {cost }}^{-}$submodel and obtain $F_{\text {cost-opt }}^{-}$under different $q_{i}$ levels. 
Step 6. Formulate $F_{\text {cost }}^{+}$submodel, including the objective function and the relevant constraints.

Step 7. Solve the $F_{\text {cost }}^{+}$submodel and obtain $F_{\text {cost-opt }}^{+}$under different $q_{i}$ levels.

Step 8. Calculate $F_{\mathrm{opt}}^{ \pm}=\left[F_{\text {cost-opt }}^{-}, F_{\text {cost-opt }}^{+}\right]$under each given $q_{i}$ level.

Step 9. Stop.

3.3. Data Collection. Consider a case wherein a manager is responsible for designing and managing a hydraulic system (as shown in Figure 2). The problem to be resolved is how to keep contamination level by allocating various filters with a minimal sum of capital to achieve a maximal system safe. The planning time period is one year. The flow rate is approximately $100 \mathrm{~L} / \mathrm{min}$, the maximal pressure is around $35 \mathrm{MPa}$, and the fluid volume of the reservoir is about $1300 \mathrm{~L}$. Table 1 shows seven different practical combinations under each scenario at low, medium, and high ingression/generation level. Table 2 presents the diameter intervals of contaminant particles (according to piecewise linearization approach), average diameters, and its corresponding initial contamination levels and contamination tolerance levels which are listed under four probability distributions. The initial contamination level of the suction line is considered to meet the cleanliness class requirement of NAS 1638 Level 7 9 [8]. It is indicated that the contaminant ingression/generation rates and the tolerance levels of the main hydraulic components are both expressed as interval values.

In addition, the average density of contaminant particles $(\rho)$ is approximately $0.5 \times 10^{3} \mathrm{~kg} / \mathrm{m}^{3}$. Table 3 provides the types and performance parameters of the filters. It is indicated that each filter possesses its own contaminant retaining capacities that are various among four different probability distributions. The cost for installing/maintaining/replacing filters and the economic losses denoted by their corresponding mean values due to maintaining/replacing filters are presented in Table $4[8,35]$.

According to NAS 1638 contamination level, the contaminant ingression/generation rates of hydraulic components are divided into low, medium, and high levels. Table 5 presents the distributions of different contaminant ingression/generation levels corresponding to different particle diameters (low level corresponding to NAS 1638 Level 1 4, medium level corresponding to Level 5 7, and high level corresponding to Level 8 10). Interactive relationships exist among a variety of impact factors and contamination-related processes, and many parameters in the studied system are uncertain. Therefore, the developed ICIP method is supposed to tackle this type of contamination control management problem.

3.4. Result Analysis. The above models can be solved through software package LINGO. Representative outcomes (as shown in Tables 6 to 8) are explicated to demonstrate important findings. Since it is a one-year plan, an estimated
TABle 1: Practicable filter combinations.

\begin{tabular}{lccccccc}
\hline Filter & \multicolumn{7}{c}{ Scenario } \\
& 1 & 2 & 3 & 4 & 5 & 6 & 7 \\
\hline$F_{1}$ & 0 & 0 & 0 & 1 & 1 & 1 & 1 \\
$F_{2}$ & 0 & 1 & 1 & 0 & 0 & 1 & 1 \\
$F_{3}$ & 1 & 0 & 1 & 0 & 1 & 0 & 1 \\
$F_{4}$ & 1 & 0 & 0 & 1 & 1 & 0 & 0 \\
Code & 0011 & 0100 & 0110 & 1001 & 1011 & 1100 & 1110 \\
\hline
\end{tabular}

replacement period was offered according to final contamination level of the hydraulic oil when the actual replacement period is longer than one year.

Tables 6(a)-6(d) show results at low ingression/generation level. Optimized system costs correspond with four probabilities. That is because none of those filters call for any replacement with four probabilities. Optimized system costs have achieved the bottom values, which means they cannot be lower any more, but there are still some tiny differences among results with four different probabilities. Take Scenario 1, for example; four results are [376.3, 382.2], [376.5, 382.8], $[377.3,385.3]$, and $[392.5,404.0]$ days corresponding to four probabilities. The solutions indicate that the system costs would decrease with $p_{i}$, where $p_{i}=0.10,0.15,0.20,0.30$ $(i=1,2,3,4)$, respectively. The replacement periods would become longer as $p_{i}$ increases $\left(p_{1}<p_{2}<p_{3}<p_{4}\right)$. In fact, an increased $p_{i}$ means more relaxed constraints, such as contaminant retaining capacity of filters and component contaminant sensitivity, and thus a raised risk in violating the two constrains.

Tables 7(a)-7(d) show results at medium ingression/ generation level. It is obvious that these results are interrupted by occasionally some bad results, the values of which do not agree with normal tendency. For example, the results under Scenario 2 are [178, 187], [166, 357.7], [175, 358.1], and $[87,360.8]$ days. The general trend is that replacement periods go up as $p_{i}$ increases except that 178 days with $p_{i}$ distribution is not on the track. However, the data still represents the principle perfectly. The same phenomenon also appears under other scenarios.

Tables $8(a)-8(d)$ show results at high ingression/generation level. Since the contamination/generation level is high, any change in $p_{i}$ would yield different contaminant retaining capacities of filters and component contaminant sensitivities and thus obviously result in different system costs. Under a probability level of $p_{i}=p_{1}=0$, the violation of the system cost and replacement period would be zero. The system costs under Scenarios 1, 2, 3, 5, 6, and 7 are RMB $¥[44500,50150]$, [59000,67400], [49950, 56550], [41600, 46850], [38250, 39750], and [42250, 47800], respectively. Under a significance level of $p_{i}=p_{2}$, the system costs under Scenarios 1, 2, 3, 5, 6 , and 7 are RMB $¥[52150,59150],[50750,57950],[45000$, 51350], [50600, 55100], [36350, 35700], and [40600, 43200], respectively. The costs are a little lower than those under the probability of $p_{1}$ due to a slightly increased risk. Then $p_{i}$ rises up to $p_{3}$ and the corresponding costs go down accordingly. Under a probability level of $p_{i}=p_{3}$, the system costs under Scenarios 1, 2, 3, 5, 6, and 7 are RMB $¥[49450,58000]$, 
TABLE 2: Particle diameters, initial contamination, and contamination tolerance levels.

\begin{tabular}{|c|c|c|c|c|c|c|c|c|c|}
\hline Contaminant particle divisions & 1 & 2 & 3 & 4 & 5 & 6 & 7 & 8 & 9 \\
\hline Range of diameters $(\mu \mathrm{m})$ & $0 \sim 5$ & $5 \sim 10$ & $10 \sim 15$ & $15 \sim 20$ & $20 \sim 25$ & $25 \sim 30$ & $30 \sim 50$ & $50 \sim 100$ & $100 \sim$ \\
\hline $\begin{array}{l}\text { Initial contamination level } N_{01 \mathrm{~m}}^{ \pm} \\
\text {(number of particles/mL) }\end{array}$ & {$[1000,1500]$} & {$[300,500]$} & {$[150,250]$} & {$[60,100]$} & {$[30,50]$} & {$[10,15]$} & {$[6,10]$} & {$[2.5,4.0]$} & {$[0.5,0.8]$} \\
\hline \multicolumn{10}{|l|}{$\begin{array}{l}\text { Contamination tolerance level } \\
S_{1 \mathrm{~nm}}^{ \pm}(\text {number of particles/mL) }\end{array}$} \\
\hline$q_{1}$ & {$[4000,6000]$} & {$[1200,2000]$} & {$[600,1000]$} & {$[250,400]$} & {$[140,230]$} & {$[50,67]$} & {$[30,43]$} & {$[12,17]$} & {$[5,7]$} \\
\hline$q_{2}$ & {$[4300,6300]$} & {$[1300,2100]$} & {$[680,1080]$} & {$[300,460]$} & {$[120,200]$} & {$[40,60]$} & {$[25,40]$} & {$[10,15]$} & {$[4,6]$} \\
\hline$q_{3}$ & {$[4600,6500]$} & {$[1500,2200]$} & {$[720,1200]$} & {$[350,530]$} & {$[160,260]$} & {$[60,71]$} & {$[35,45]$} & {$[14,19]$} & {$[6,8]$} \\
\hline$q_{4}$ & {$[8000,12000]$} & {$[2400,4000]$} & {$[1200,2000]$} & {$[500,800]$} & {$[240,400]$} & {$[80,120]$} & {$[50,80]$} & {$[20,30]$} & {$[8,12]$} \\
\hline \multicolumn{10}{|l|}{$\begin{array}{l}\text { Contamination tolerance level } \\
S_{2 \mathrm{~nm}}^{ \pm}(\text {number of particles } / \mathrm{mL})\end{array}$} \\
\hline$q_{1}$ & {$[2000,3000]$} & {$[600,1000]$} & {$[300,500]$} & {$[120,200]$} & {$[60,100]$} & {$[20,30]$} & {$[12,20]$} & {$[5,8]$} & {$[2,3]$} \\
\hline$q_{2}$ & {$[2100,3100]$} & {$[700,1200]$} & {$[380,560]$} & {$[150,210]$} & {$[70,105]$} & {$[30,35]$} & {$[16,23]$} & {$[7,10]$} & {$[3,4]$} \\
\hline$q_{3}$ & {$[2200,3200]$} & {$[800,1280]$} & {$[450,630]$} & {$[180,220]$} & {$[80,110]$} & {$[40,40]$} & {$[20,26]$} & {$[9,12]$} & {$[4,5]$} \\
\hline$q_{4}$ & {$[4000,6000]$} & {$[1200,2000]$} & {$[600,1000]$} & {$[240,400]$} & {$[120,200]$} & {$[40,60]$} & {$[24,40]$} & {$[10,16]$} & {$[4,6]$} \\
\hline
\end{tabular}

TABLE 3: Type and performance parameters of filters.

\begin{tabular}{|c|c|c|c|c|c|c|c|c|}
\hline \multirow{2}{*}{ Filters } & \multirow{2}{*}{ Type of filters } & \multirow{2}{*}{$\begin{array}{l}\text { Fineness of } \\
\text { filters }(\mu \mathrm{m})\end{array}$} & \multirow{2}{*}{$\begin{array}{l}\text { Rated flow } \\
\text { (L/min) }\end{array}$} & \multirow{2}{*}{$\begin{array}{c}\text { Maximum } \\
\text { pressure }(\mathrm{MPa})\end{array}$} & \multicolumn{4}{|c|}{ Contaminant-holding capability $C_{k}(\mathrm{~g})$} \\
\hline & & & & & $p_{1}$ & $p_{2}$ & $p_{3}$ & $p_{4}$ \\
\hline Suction filter & AS 150-01 & 100 & 200 & Low & {$[100,120]$} & {$[105,125]$} & {$[110,130]$} & {$[200,240]$} \\
\hline Pressure filter & HD 414-56 & 10 & 210 & 35 & {$[50,60]$} & {$[60,71]$} & {$[65,75]$} & {$[100,120]$} \\
\hline Return filter & E 211-58 & 20 & 210 & Low & {$[70,85]$} & {$[72,86]$} & {$[80,90]$} & {$[140,170]$} \\
\hline Bypass filter & FNA 016-553 & $1 \mathrm{E}$ & 16 & 0.4 & {$[65,80]$} & {$[67,82]$} & {$[72,86]$} & {$[130,160]$} \\
\hline
\end{tabular}

TABLe 4: Cost data.

\begin{tabular}{lcccc}
\hline Filters & $\begin{array}{c}\text { Price of filters } B_{k} \\
(\text { RMB } ¥)\end{array}$ & $\begin{array}{c}\text { Replacement } \\
\text { expenditure } E_{k}(\text { RMB } ¥)\end{array}$ & $\begin{array}{c}\text { Wage of maintenance } \\
\text { worker } M_{k}(\text { RMB } ¥) \\
\text { per time }\end{array}$ & $\begin{array}{c}\text { Downtime cost } P_{k} \\
(\text { RMB } ¥) \text { per time }\end{array}$ \\
\hline Suction filter & {$[1000,1300]$} & {$[500,600]$} & 50 & 200 \\
Pressure filter & {$[8000,9500]$} & {$[1100,1400]$} & 50 & 200 \\
Return filter & {$[2000,2500]$} & {$[900,1100]$} & 50 & 200 \\
Bypass filter & 9000 & 1300 & 50 & 200 \\
\hline
\end{tabular}

TABLE 5: Initial contaminant ingression/generation rates of hydraulic components.

\begin{tabular}{|c|c|c|c|c|c|c|c|c|c|}
\hline $\begin{array}{l}\text { Contaminant particle } \\
\text { divisions }\end{array}$ & 1 & 2 & 3 & 4 & 5 & 6 & 7 & 8 & 9 \\
\hline & \multicolumn{9}{|c|}{ Low level $\left(\times 10^{3}\right.$ number of particles/min) } \\
\hline$R_{1 \mathrm{~nm}}^{ \pm}$ & {$[25,35]$} & {$[7,8]$} & {$[2.5,3.5]$} & {$[0.7,1.6]$} & {$[0.55,0.65]$} & {$[0.18,0.3]$} & {$[0.07,0.15]$} & {$[0.03,0.09]$} & {$[0.017,0.025]$} \\
\hline$R_{2 \mathrm{~nm}}^{ \pm}$ & {$[100,400]$} & {$[28,32]$} & {$[10,14]$} & {$[2.8,6.4]$} & {$[2.2,2.6]$} & {$[0.72,1.14]$} & {$[0.28,0.6]$} & {$[0.12,0.36]$} & {$[0.068,0.1]$} \\
\hline \multirow[t]{2}{*}{$R_{3 \mathrm{~nm}}^{ \pm}$} & {$[50,70]$} & {$[14,16]$} & {$[5,7]$} & {$[1.4,3.2]$} & {$[1.1,1.3]$} & {$[0.36,0.52]$} & {$[0.14,0.2]$} & {$[0.06,0.18]$} & {$[0.034,0.05]$} \\
\hline & \multicolumn{9}{|c|}{ Medium level $\left(\times 10^{4}\right.$ number of particles $\left./ \mathrm{min}\right)$} \\
\hline$R_{1 \mathrm{~nm}}^{ \pm}$ & {$[33,36]$} & {$[9,13]$} & {$[3,7]$} & {$[1,3]$} & {$[0.5,1.5]$} & {$[0.2,0.45]$} & {$[0.1,0.25]$} & {$[0.08,0.11]$} & {$[0.007,0.027]$} \\
\hline$R_{2 \mathrm{~nm}}^{ \pm}$ & {$[132,144]$} & {$[36,52]$} & {$[12,28]$} & {$[4,12]$} & {$[2,6]$} & {$[0.8,1.8]$} & {$[0.4,1]$} & {$[0.32,0.44]$} & {$[0.028,0.108]$} \\
\hline \multirow[t]{2}{*}{$R_{3 \mathrm{~nm}}^{ \pm}$} & {$[66,72]$} & {$[18,26]$} & {$[6,14]$} & {$[2,6]$} & {$[1,3]$} & {$[0.4,0.9]$} & {$[0.2,0.5]$} & {$[0.16,0.22]$} & {$[0.014,0.054]$} \\
\hline & \multicolumn{9}{|c|}{ High level $\left(\times 10^{4}\right.$ number of particles $\left./ \mathrm{min}\right)$} \\
\hline$R_{1 \mathrm{~nm}}^{ \pm}$ & {$[255,270]$} & {$[85,100]$} & {$[40,50]$} & {$[10,20]$} & {$[7.5,8.5]$} & {$[2.5,3.5]$} & {$[1,2]$} & {$[0.5,1]$} & {$[0.1,0.2]$} \\
\hline$R_{2 \mathrm{~nm}}^{ \pm}$ & {$[1020,1080]$} & {$[340,400]$} & {$[160,200]$} & {$[40,80]$} & {$[30,32]$} & {$[10,14]$} & {$[4,8]$} & {$[2,4]$} & {$[0.4,0.8]$} \\
\hline$R_{3 \mathrm{~nm}}^{ \pm}$ & {$[510,540]$} & {$[170,200]$} & {$[80,100]$} & {$[20,40]$} & {$[15,16]$} & {$[5,7]$} & {$[2,4]$} & {$[1,2]$} & {$[0.2,0.4]$} \\
\hline
\end{tabular}


TABLE 6: (a) Results for optimized system cost and filter-replacement period under low ingression/generation rate (8 hours per day) (when $p_{1}=0.10$ ). (b) Results for optimized system cost and filter-replacement period under low ingression/generation rate ( 8 hours per day) (when $\left.p_{2}=0.15\right)$. (c) Results for optimized system cost and filter-replacement period under low ingression/generation rate ( 8 hours per day) (when $p_{3}=0.20$ ). (d) Results for optimized system cost and filter-replacement period under low ingression/generation rate (8 hours per day) (when $\left.p_{4}=0.30\right)$.

(a)

\begin{tabular}{|c|c|c|c|c|}
\hline \multirow{2}{*}{ Scenario } & \multirow{2}{*}{ Optimized system cost (RMB $¥)$} & \multicolumn{3}{|c|}{ Replacement period of filter element (days) } \\
\hline & & $k=1$ & $k=2$ & $k=3$ \\
\hline 1 & {$[2000,2500]$} & - & - & {$[376.3,382.2]$} \\
\hline 2 & {$[8000,9500]$} & - & {$[385.8,398.8]$} & - \\
\hline 3 & {$[10000,12000]$} & - & {$[413,433.5]$} & {$[403,419.1]$} \\
\hline 4 & {$[1000,1300]$} & {$[366.6,367.2]$} & - & - \\
\hline 5 & {$[3000,3800]$} & {$[384.1,390.6]$} & - & {$[379.2,385.7]$} \\
\hline 6 & {$[9000,10800]$} & {$[379.9,385.2]$} & {$[415.2 .8,415]$} & - \\
\hline 7 & {$[11000,13300]$} & {$[399.1,409.2]$} & {$[425.0,446.9]$} & {$[404.7,420.1]$} \\
\hline \multicolumn{5}{|c|}{ (b) } \\
\hline \multirow{2}{*}{ Scenario } & \multirow{2}{*}{ Optimized system cost (RMB $¥)$} & \multicolumn{3}{|c|}{ Replacement period of filter element (days) } \\
\hline & & $k=1$ & $k=2$ & $k=3$ \\
\hline 1 & {$[2000,2500]$} & - & - & {$[376.5,382.8]$} \\
\hline 2 & {$[8000,9500]$} & - & {$[392.4,408.4]$} & - \\
\hline 3 & {$[10000,12000]$} & - & {$[424.03 .1,449.4]$} & {$[404.5 .0,420.8]$} \\
\hline 4 & {$[1000,1300]$} & {$[366.7,367.3]$} & - & - \\
\hline 5 & {$[3000,3800]$} & {$[384.9,391.9]$} & - & {$[379.5,386.4]$} \\
\hline 6 & {$[9000,10800]$} & {$[380.6,386.3]$} & {$[409.6,426.3]$} & - \\
\hline 7 & {$[11000,13300]$} & {$[400.6,411.5]$} & {$[437.1,464.4]$} & {$[405.2,421.8]$} \\
\hline
\end{tabular}

(c)

\begin{tabular}{|c|c|c|c|c|}
\hline \multirow{2}{*}{ Scenario } & \multirow{2}{*}{ Optimized system cost (RMB $¥)$} & \multicolumn{3}{|c|}{ Replacement period of filter element (days) } \\
\hline & & $k=1$ & $k=2$ & $k=3$ \\
\hline 1 & {$[2000,2500]$} & - & - & {$[377.3,385.3]$} \\
\hline 2 & {$[8000,9500]$} & - & {$[394.8,413.2]$} & - \\
\hline 3 & {$[10000,12000]$} & - & {$[428.0 .0,457.3]$} & {$[406.7 .5,427.8]$} \\
\hline 4 & {$[1000,1300]$} & {$[366.8,367.5]$} & - & - \\
\hline 5 & {$[3000,3800]$} & {$[385.8,393.2]$} & - & {$[380.3,389.2]$} \\
\hline 6 & {$[9000,10800]$} & {$[381.3,387.4]$} & {$[412.4,431.8]$} & - \\
\hline 7 & {$[11000,13300]$} & {$[402.1,413.8]$} & {$[441.5,473.2]$} & {$[407.5,428.9]$} \\
\hline \multicolumn{5}{|c|}{ (d) } \\
\hline \multirow{2}{*}{ Scenario } & \multirow{2}{*}{ Optimized system cost (RMB ¥) } & \multicolumn{3}{|c|}{ Replacement period of filter element (days) } \\
\hline & & $k=1$ & $k=2$ & $k=3$ \\
\hline 1 & {$[2000,2500]$} & - & - & {$[392.5,404.0]$} \\
\hline 2 & {$[8000,9500]$} & - & {$[422.0,446.6]$} & - \\
\hline 3 & {$[10000,12000]$} & - & {$[472.8 .0,512.8]$} & {$[450.6 .7,480.0]$} \\
\hline 4 & {$[1000,1300]$} & {$[368.6,367.7]$} & - & - \\
\hline 5 & {$[3000,3800]$} & {$[404.3,395.9]$} & - & {$[397.7,410.2]$} \\
\hline 6 & {$[9000,10800]$} & {$[397.3,389.7]$} & {$[444.2,470.7]$} & - \\
\hline 7 & {$[11000,13300]$} & {$[435.0,418.4]$} & {$[491.2,534.6]$} & {$[452.1,482.0]$} \\
\hline
\end{tabular}


TABLE 7: (a) Results for optimized system cost and filter-replacement period under medium ingression/generation rate (8 hours per day) (when $\left.p_{1}=0.10\right)$. (b) Results for optimized system cost and filter-replacement period under medium ingression/generation rate ( 8 hours per day) (when $\left.p_{2}=0.15\right)$. (c) Results for optimized system cost and filter-replacement period under medium ingression/generation rate (8 hours per day) (when $p_{3}=0.20$ ). (d) Results for optimized system cost and filter-replacement period under medium ingression/generation rate ( 8 hours per day) (when $p_{4}=0.30$ ).

(a)

\begin{tabular}{|c|c|c|c|c|}
\hline \multirow{2}{*}{ Scenario } & \multirow{2}{*}{ Optimized system cost (RMB $¥)$} & \multicolumn{3}{|c|}{ Replacement period of filter element (days) } \\
\hline & & $k=1$ & $k=2$ & $k=3$ \\
\hline 1 & {$[5200,6600]$} & - & - & {$[361.3,362.9]$} \\
\hline 2 & {$[14450,14750]$} & - & {$[178,187]$} & - \\
\hline 3 & {$[15000,15000]$} & - & {$[358.1,362.3]$} & {$[192,244]$} \\
\hline 4 & {$[2150,2500]$} & {$[178,187]$} & - & - \\
\hline 5 & {$[5150,6450]$} & {$[365.4,366.4]$} & - & {$[362.1,363.7]$} \\
\hline 6 & {$[12450,13300]$} & {$[128,274]$} & {$[147,253]$} & - \\
\hline 7 & {$[14650,14650]$} & {$[194,367.5]$} & {$[363.1,366.8]$} & {$[232,361.2]$} \\
\hline
\end{tabular}

(b)

\begin{tabular}{lcccc}
\hline Scenario & Optimized system cost (RMB ¥) & \multicolumn{3}{c}{ Replacement period of filter element (days) } \\
& & $k=1$ & $k=2$ & $k=3$ \\
1 & {$[5200,6600]$} & - & - & {$[67,256]$} \\
2 & {$[12800,13400]$} & - & {$[166,357.7]$} & {$[247,364.2]$} \\
3 & {$[15000,15000]$} & {$[231,364.9]$} & - & - \\
4 & {$[2150,2500]$} & {$[174,186]$} & - & {$[362.1,363.7]$} \\
5 & {$[5150,6450]$} & {$[364.3,365.1]$} & {$[151,365.5]$} & - \\
7 & {$[10800,11100]$} & {$[230,367.6]$} & {$[239,364.0]$} & {$[124,364.2]$} \\
\hline
\end{tabular}

(c)

\begin{tabular}{|c|c|c|c|c|}
\hline \multirow{2}{*}{ Scenario } & \multirow{2}{*}{ Optimized system cost (RMB $¥)$} & \multicolumn{3}{|c|}{ Replacement period of filter element (days) } \\
\hline & & $k=1$ & $k=2$ & $k=3$ \\
\hline 1 & {$[3850,5450]$} & - & - & {$[70,142]$} \\
\hline 2 & {$[12800,13400]$} & - & {$[175,358.1]$} & - \\
\hline 3 & {$[15000,15000]$} & - & {$[127,364.2]$} & {$[361.4,364.7]$} \\
\hline 4 & {$[2150,2500]$} & {$[202,364.9]$} & - & - \\
\hline 5 & {$[5150,6450]$} & {$[186,365.5]$} & - & {$[91,363.9]$} \\
\hline 6 & {$[10800,11100]$} & {$[364.3,365.2]$} & {$[159,366.0]$} & - \\
\hline 7 & {$[14650,14650]$} & {$[365.9,367.8]$} & {$[260,368.9]$} & {$[130,265]$} \\
\hline
\end{tabular}

(d)

\begin{tabular}{|c|c|c|c|c|}
\hline \multirow{2}{*}{ Scenario } & \multirow{2}{*}{ Optimized system cost (RMB $¥)$} & \multicolumn{3}{|c|}{ Replacement period of filter element (days) } \\
\hline & & $k=1$ & $k=2$ & $k=3$ \\
\hline 1 & {$[3850,4300]$} & - & - & {$[362.5,364.6]$} \\
\hline 2 & {$[11150,10700]$} & - & {$[187,360.8]$} & - \\
\hline 3 & {$[12000,12500]$} & - & {$[244,369.0]$} & {$[187,364.9]$} \\
\hline 4 & {$[1750,2150]$} & {$[187,364.9]$} & - & - \\
\hline 5 & {$[3800,4150]$} & {$[366.7,366.8]$} & - & {$[363.4,365.6]$} \\
\hline 6 & {$[10800,10350]$} & {$[365.1,365.4]$} & {$[364.6,369.1]$} & - \\
\hline 7 & {$[12150,13300]$} & {$[246,368.2]$} & {$[373.8,367.7]$} & {$[364.3,369.1]$} \\
\hline
\end{tabular}


TABLE 8: (a) Results for optimized system cost and filter-replacement period under high ingression/generation rate (8 hours per day) (when $\left.p_{1}=0.10\right)$. (b) Results for optimized system cost and filter-replacement period under high ingression/generation rate (8 hours per day) (when $p_{2}=0.15$ ). (c) Results for optimized system cost and filter-replacement period under high ingression/generation rate ( 8 hours per day) (when $\left.p_{3}=0.20\right)$. (d) Results for optimized system cost and filter-replacement period under high ingression/generation rate (8 hours per day) (when $p_{4}=0.30$ ).

(a)

\begin{tabular}{|c|c|c|c|c|c|}
\hline \multirow{2}{*}{ Scenario } & \multirow{2}{*}{ Optimized system cost (RMB $¥)$} & \multicolumn{4}{|c|}{ Replacement period of filter element (days) } \\
\hline & & $k=1$ & $k=2$ & $k=3$ & $k=4$ \\
\hline 1 & {$[53500,59150]$} & - & - & {$[12,17]$} & {$[98,105]$} \\
\hline 2 & {$[59000,67400]$} & & {$[7,9]$} & & \\
\hline 3 & {$[49950,56550]$} & & {$[17,47]$} & {$[26,27]$} & \\
\hline 4 & - & - & & & - \\
\hline 5 & {$[50600,55850]$} & {$[71,110]$} & & {$[16,22]$} & {$[107,114]$} \\
\hline 6 & {$[38250,39750]$} & {$[19,88]$} & {$[19,46]$} & & \\
\hline 7 & {$[42250,47800]$} & {$[24,33]$} & {$[17,47]$} & {$[27,70]$} & \\
\hline
\end{tabular}

(b)

\begin{tabular}{|c|c|c|c|c|c|}
\hline \multirow{2}{*}{ Scenario } & \multirow{2}{*}{ Optimized system cost (RMB $¥)$} & \multicolumn{4}{|c|}{ Replacement period of filter element (days) } \\
\hline & & $k=1$ & $k=2$ & $k=3$ & $k=4$ \\
\hline 1 & {$[52150,59150]$} & - & - & {$[12,18]$} & {$[101,108]$} \\
\hline 2 & {$[50750,57950]$} & & {$[22,22]$} & & \\
\hline 3 & {$[45000,51350]$} & & {$[7,17]$} & {$[48,69]$} & \\
\hline 4 & - & - & & & - \\
\hline 5 & {$[50600,55100]$} & {$[74,115]$} & & {$[16,23]$} & {$[110,117]$} \\
\hline 6 & {$[36350,35700]$} & {$[26,65]$} & {$[13,22]$} & & \\
\hline 7 & {$[40600,43200]$} & {$[32,88]$} & {$[17,269]$} & {$[42,49]$} & \\
\hline
\end{tabular}

(c)

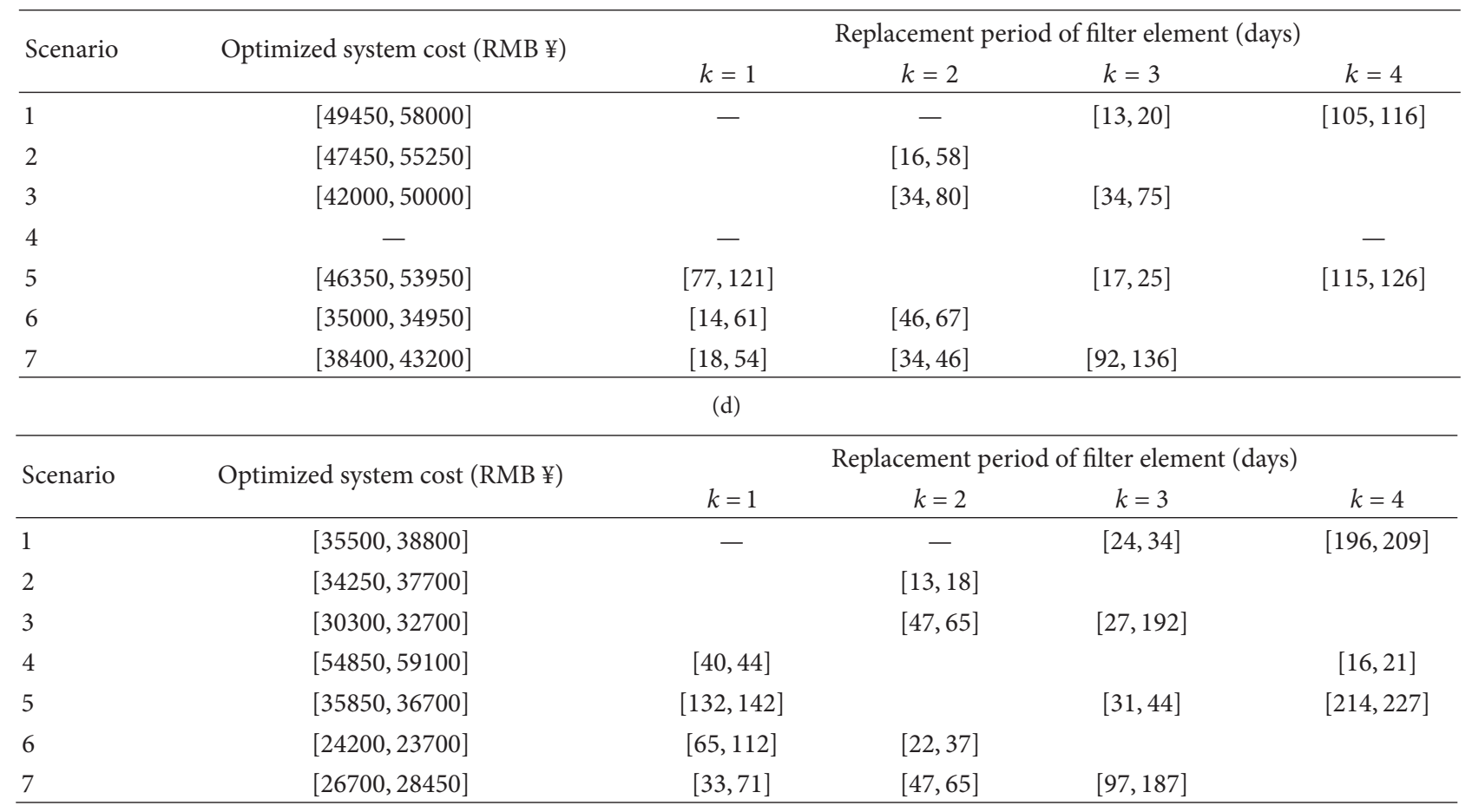


, [42000, 50000], [46350, 53950], [35000, 34950], and [38400, 43200], respectively. Under a probability level of $p_{i}=p_{4}$, the system risks the most while the costs of the system prominently fall. The system costs under seven scenarios are RMB $¥[35500,38800],[34250,37700],[30300,32700],[54850$, 59100], [35850, 36700], [24200, 23700], and [26700, 28450], respectively.

Figure 3 presents outcomes for system optimal cost of all scenarios under low contaminant ingression/generation rates. When contaminant ingression/generation rate is low, the system maintenance cost achieves the optimal one. Accordingly, results under four probabilities denote the general trend and some tiny differences among results with different probabilities. Obviously, the system shows good characteristic of resisting violation.

Figure 4 presents outcomes for system optimal cost of all scenarios under medium contaminant ingression/generation rates under four probabilities. Take scenario 001, for example; the cost under probability $p_{1}$ is 0 less than that under probability $p_{2}$; the cost under probability $p_{2}$ is $[1150,1350]$ less than that under probability $p_{3}$; the cost under probability $p_{3}$ is $\operatorname{RMB} ¥[0,1150]$ less than that under probability $p_{4}$. All the increments are small which means the system is good at resisting risk. Take scenario 010, for example; the cost under probability $p_{1}$ is RMB $¥[1350,1650]$ less than that under probability $p_{2}$; the cost under probability $p_{2}$ is 0 less than that under probability $p_{3}$; the cost under probability $p_{3}$ is RMB $¥[2100,2250]$ less than that under probability $p_{4}$. Small increments show good risk-resisting ability. Take scenario 011, for example; the cost under probability $p_{1}$ is 0 less than that under probability $p_{2}$; the cost under probability $p_{2}$ is 0 less than that under probability $p_{3}$; the cost under probability $p_{3}$ is $\mathrm{RMB} ¥[2500,3000]$ less than that under probability $p_{4}$. Environment violation has little influence on cost change. Take scenario 100, for example; the cost under probability $p_{1}$ is 0 less than that under probability $p_{2}$; the cost under probability $p_{2}$ is 0 less than that under probability $p_{3}$; the cost under probability $p_{3}$ is $\mathrm{RMB} ¥[350,400]$ less than that under probability $p_{4}$. Under scenario 101 , the cost under probability $p_{1}$ is 0 less than that under probability $p_{2}$; the cost under probability $p_{2}$ is 0 less than that under probability $p_{3}$; the cost under probability $p_{3}$ is $\mathrm{RMB} ¥[1350,2300]$ less than that under probability $p_{4}$. Under scenario 110 , the cost under probability $p_{1}$ is RMB $¥[1650,2200]$ less than that under probability $p_{2}$; the cost under probability $p_{2}$ is 0 less than that under probability $p_{3}$; the cost under probability $p_{3}$ is RMB $¥$ $[300,450]$ less than that under probability $p_{4}$. Under scenario 111 , the cost under probability $p_{1}$ is 0 less than that under probability $p_{2}$; the cost under probability $p_{2}$ is 0 less than that under probability $p_{3}$; the cost under probability $p_{3}$ is RMB $¥$ $[1350,2500]$ less than that under probability $p_{4}$.

Figure 5 presents outcomes for system optimal cost of all scenarios under high contaminant ingression/generation rates under four probabilities. Take scenario 001, for example; the cost under probability $p_{1}$ is $\mathrm{RMB} ¥[0,1350]$ less than that under probability $p_{2}$; the cost under probability $p_{2}$ is RMB $¥[-5100,2700]$ less than that under probability $p_{3}$; the cost under probability $p_{3}$ is $[13950,25450]$ less than that under probability $p_{4}$. All the increments are large which means the

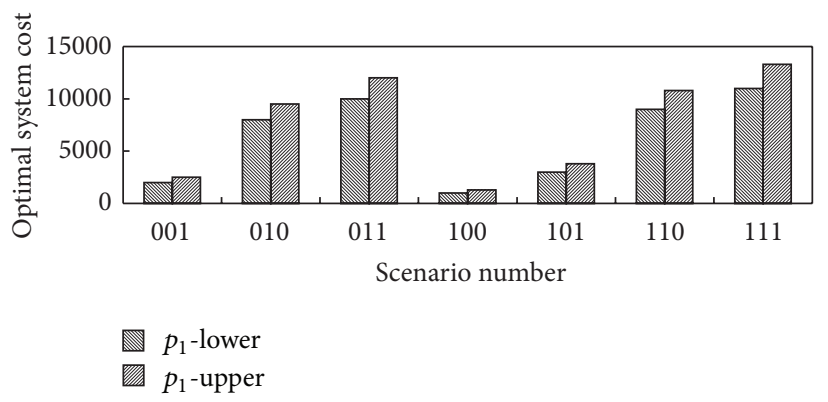

FIGURE 3: Optimal costs under low contaminant ingression/generation level.

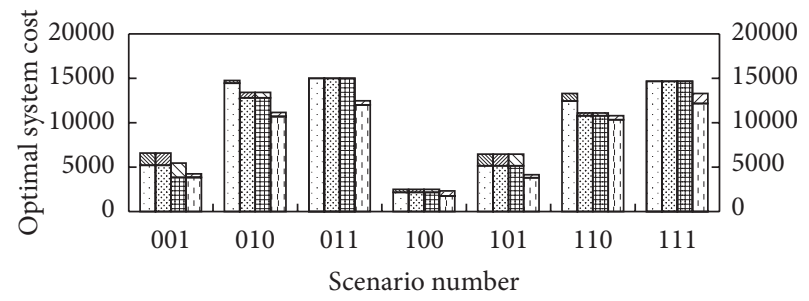

\begin{tabular}{|c|c|}
\hline $\mathbb{Q} p_{1}$-upper & $\triangle p_{3}$-upper \\
\hline$p_{2}$-lower & 四 $p_{4}$-lower \\
\hline$\square p_{2}$-upper & $\square p_{4}$-upper \\
\hline 囲 $p_{3}$-lower & $p_{1}$-lower \\
\hline
\end{tabular}

FIgURE 4: Optimal costs under medium contaminant ingression/ generation level.

system is bad at resisting risk. Take scenario 010, for example; the cost under probability $p_{1}$ is RMB $¥[8250,9450]$ less than that under probability $p_{2}$; the cost under probability $p_{2}$ is $\operatorname{RMB} ¥[3300,2700]$ less than that under probability $p_{3}$; the cost under probability $p_{3}$ is RMB $¥[13200,17550]$ less than that under probability $p_{4}$. Small increments show bad risk-resisting ability. Take scenario 011, for example; the cost under probability $p_{1}$ is RMB $¥[50,5200]$ less than that under probability $p_{2}$; the cost under probability $p_{2}$ is RMB $¥[1350$, 3000 ] less than that under probability $p_{3}$; the cost under probability $p_{3}$ is RMB $¥[11700,17300]$ less than that under probability $p_{4}$. Environment violation has great influence on cost changes. Results under scenario 100 are infeasible except for that under probability $p_{4}$ because of severe violation. Under scenario 101, the cost under probability $p_{1}$ is $[0,750]$ less than that under probability $p_{2}$; the cost under probability $p_{2}$ is RMB $¥[1150,4250]$ less than that under probability $p_{3}$; the cost under probability $p_{3}$ is RMB $¥[10500,17250]$ less than that under probability $p_{4}$. Under scenario 110 , the cost under probability $p_{1}$ is $\mathrm{RMB} ¥[2550,3400]$ less than that under probability $p_{2}$; the cost under probability $p_{2}$ is RMB $¥$ $[750,1350]$ less than that under probability $p_{3}$; the cost under probability $p_{3}$ is RMB $¥[10800,11250]$ less than that under probability $p_{4}$. Under scenario 111 , the cost under probability $p_{1}$ is RMB $¥[1650,4600]$ less than that under probability $p_{2}$; the cost under probability $p_{2}$ is RMB $¥[0,2200]$ less than that under probability $p_{3}$; the cost under probability $p_{3}$ is RMB $¥$ $[11700,14750]$ less than that under probability $p_{4}$. Generally 


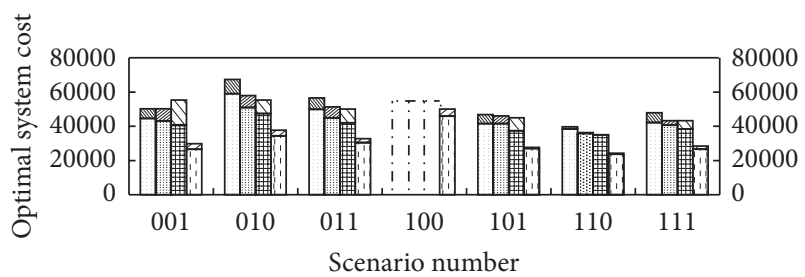

$\begin{array}{ll}\square p_{1} \text {-upper } & \square p_{3} \text {-upper } \\ 0 p_{2} \text {-lower } & \text { 国 } p_{4} \text {-lower } \\ \square p_{2} \text {-upper } & \square p_{4} \text {-upper } \\ \text { 四 } p_{3} \text {-lower } & \square p_{1} \text {-lower }\end{array}$

Figure 5: Optimal costs under high contaminant ingression/generation level.

speaking, when contamination ingression/generation rate is high, the hydraulic system is more vulnerable to any violation from environment.

The $p_{i}$ levels represent a set of probabilities at which the constraints will be violated (i.e., the admissible risk of violating the constraints). Thus, the relation between $F^{ \pm}$and $p_{i}$ would demonstrate a tradeoff between system cost and constraint-violation risk. An increased $p_{i}$ means a raised risk of constraint violation and, at the same time, it will lead to a decreased strictness for the constraints and thus a decreased system cost. Such a decreased cost, however, would be linked to a potentially increased threat of component failure and thus a raised risk of constraint violation. Figures 3, 4, 5, $6,7,8,9,10$, and 11 indicate that, as the actual values of the decision variables vary within their two bounds, the expected system cost will change correspondingly between $F_{\text {cost-opt }}^{-}$and $F_{\text {cost-opt }}^{+}$with different reliability levels. Decisions at a lower $p_{i}$ level would lead to an increased reliability in fulfilling the system requirements but with a higher cost; in comparison, decisions at a higher $p_{i}$ level would result in a lower cost, but the risk of violating the constraints would be increased. These demonstrate a tradeoff between the filter maintenance cost and the system-failure risk due to the dual uncertainties that exist in various system components (i.e., interval and probabilistic information). In practice, planning with a higher system cost may guarantee that hydraulic system requirements and economic budget are met with higher system reliability; however, when the plan aims towards a lower system cost, these requirements may not be adequately met because of higher system risk.

3.5. Discussion. Solutions of the inexact chance-constrained integer linear programming (ICIP) model (Tables 6(a), 7(a), and $8(\mathrm{a}))$ provide two extremes of the expected system cost. In practice, decisions for a lower cost may correspond to advantageous system conditions (e.g., lower contaminated ingression/generation rate), while those with a higher cost correspond to more demanding conditions. In comparison with the interval-fuzzy chance-constrained integer programming (IFCIP) method, the ICIP does not integrate the fuzzy programming within a general framework. Accordingly, the

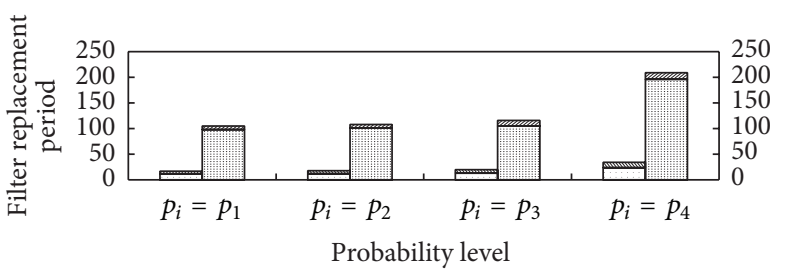

$\begin{array}{lll}\mathbb{Q} \text { Filter } 3 \text { (upper) } & \text { Filter } 4 \text { (upper) } \\ \square \text { Filter } 3 \text { (lower) } & \square \text { Filter } 4 \text { (lower) }\end{array}$

FIgURE 6: Filter replacement periods for Scenario 1 under high contaminant ingression/generation level.

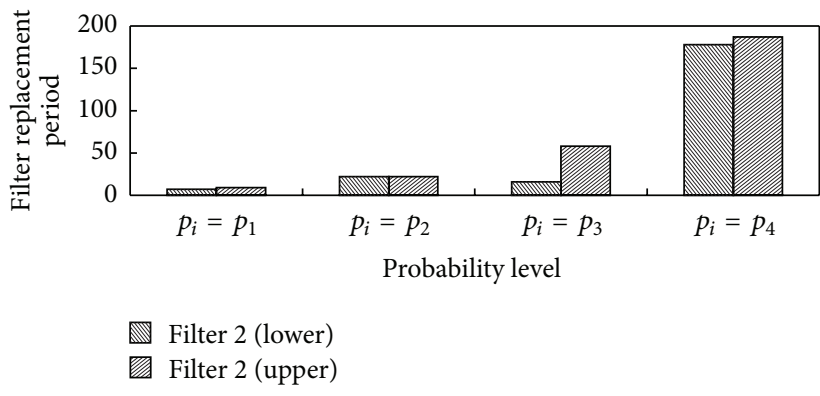

Figure 7: Filter replacement periods for Scenario 2 under high contaminant ingression/generation level.

analytical approaches have many differences between the two methods.

The interval-parameter two-stage stochastic nonlinear programming (ITSNP) is a hybrid methodology of inexact optimization and two-stage stochastic programming. Thus, the method has some advantages over other approaches, such as the following. (a) It can deal with uncertainties that exist in FPS through generating scenarios of its future events; these scenarios correspond to different effects of varying filter-allocations on the economic objective. (b) It can reflect the dynamics of system uncertainties and decision processes under different scenarios. In comparison with the ICIP, the ITSNP has the following limitations: (i) it can only generate one interval solution without information about the risk of violating the capacity constraints; (ii) the system cost obtained through the ITSNP model is generally higher than those through the ICIP method (under a range of $p_{i}$ levels) since no relaxation on capacity constraints is allowed in the ITSNP [39]. Generally, without the chance constraints, the ITSNP is unable to support in-depth analysis of the tradeoff between system cost and system-failure risk. It may potentially result in system failure and thus increased system costs. The problem can be solved through a chance-constrained linear programming method by letting all left-hand-side interval coefficients (including the cost coefficients) in the ICIP model be equal to their midvalues. The system costs from the solutions of chance-constrained linear programming (CLP) lie within the ICIP solution intervals, demonstrating the stability of the ICIP solutions. With the CLP, only one deterministic solution corresponding to each $p_{i}$ level is generated, since the model's left-hand-side 


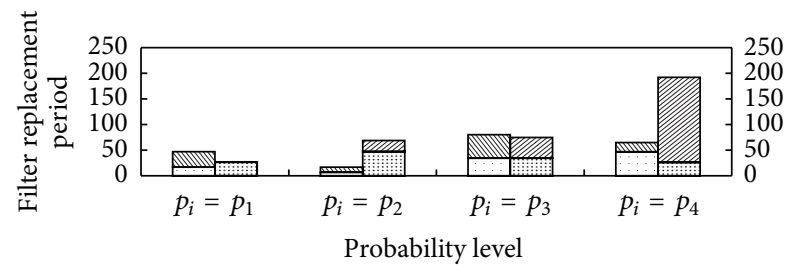

$\begin{array}{ll}\mathbb{Q} \text { Filter } 2 \text { (upper) } & \text { Filter } 3 \text { (upper) } \\ \text { Filter } 2 \text { (lower) } & \text { Filter } 3 \text { (lower) }\end{array}$

FIGURE 8: Filter replacement periods for Scenario 3 under high contaminant ingression/generation level.
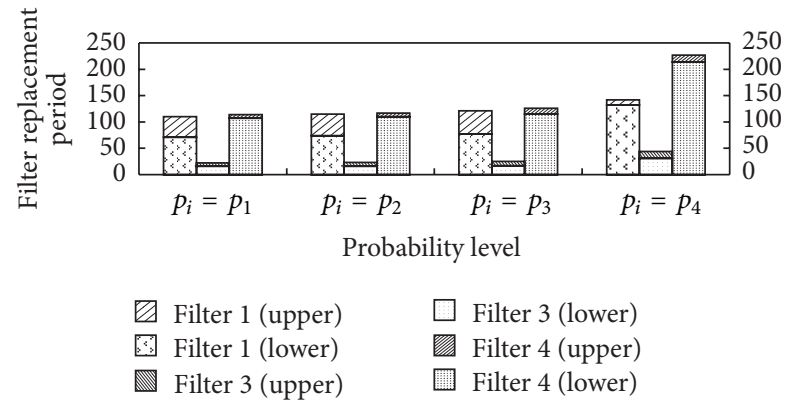

FIGURE 9: Filter replacement periods for Scenario 5 under high contaminant ingression/generation level.

coefficients are all assumed to be deterministic. However, the sensitivity analysis can only provide an individual response to variations of the uncertain inputs and, thus, can hardly reflect interactions among various uncertain parameters. Therefore, in comparison with the CLP, the ICIP method can incorporate more uncertain information within its modeling framework. The obtained interval solutions under different risk levels of violating the capacity constraints can be used to generate decision alternatives and help hydraulic system managers to identify desired policies under various condition, economic, and system-reliability constraints.

\section{Conclusions}

An inexact chance-constrained integer programming (ICIP) method has been developed for contaminant control for hydraulic system. The method improves upon the existing interval-integer and chance-constrained programming approaches by allowing uncertainties presented as both probability distributions and discrete intervals to be effectively incorporated within the optimization framework. Moreover, it can help examine the reliability of satisfying (or risk of violating) system constraints under uncertainty.

The developed method has been applied to a case of hydraulic system operation. Violations for capacity constraints are allowed under a range of significance levels. Interval solutions associated with different risk levels of constraint violation have been obtained. They were used for generating decision alternatives and thus help waste managers to identify

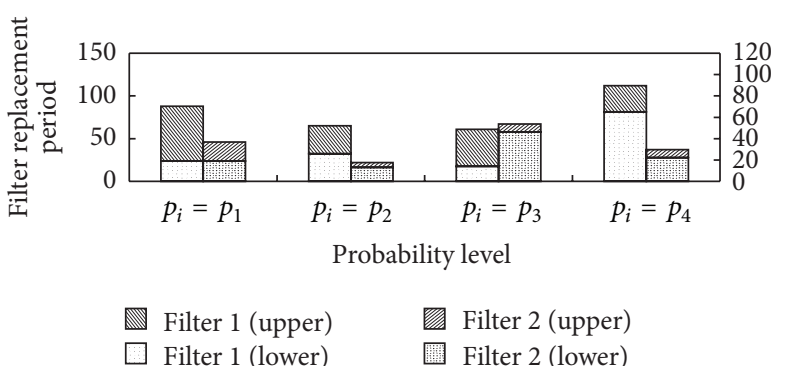

FIGURE 10: Filter replacement periods for Scenario 6 under high contaminant ingression/generation level.
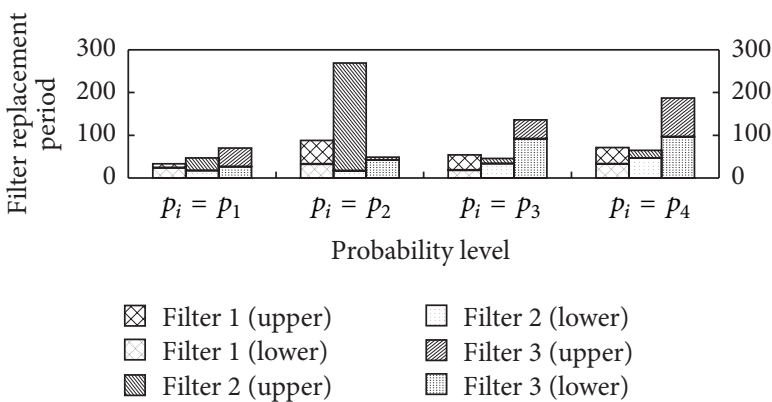

FIGURE 11: Filter replacement periods for Scenario 7 under high contaminant ingression/generation level.

desired policies under various environmental, economic, and system-reliability constraints.

The ICIP can incorporate more uncertain information within its modeling framework. It can reflect economic penalties as corrective measures or recourse against any infeasibilities arising due to a particular realization of uncertainty. Thus, the method provides not only decision variable solutions presented as stable intervals but also the associated risk levels in violating the system constraints. It can therefore support an in-depth analysis of the tradeoff between system cost and system-failure risk.

Although this study is the first attempt for contamination control of hydraulic system through developing the ICIP approach, the results suggest that this integrated technique is applicable to other hydraulic contamination control problems that involve uncertainties presented in multiple formats.

\section{Nomenclature}

\footnotetext{
“_"/“": Superscripts represent lower- and upper-bounds of the interval parameters, respectively

$\beta_{k m}$ : $\quad$ Filtration ratio of filter $k$ for a given particle diameter range $m$ ( $k=1$ for the suction filter, $k=2$ for the pressure filter, $k=3$ for the return filter, and $k=4$ for the bypass filter)

$\rho: \quad$ Average density of contaminants $\left(\mathrm{kg} / \mathrm{m}^{3}\right)$

$\lambda^{ \pm}$: $\quad$ Degree of satisfaction for the fuzzy objective and/or constraints
} 
$A_{k n}: \quad$ Binary decision variable, if filter $k$ needs to be replaced in period $n$, then $A_{k n}=1$; otherwise $A_{k n}=0$

$B_{k}: \quad$ Purchase price of filter $k(\mathrm{RMB} ¥, k=1,2,3)$

$C_{k}$ : Characteristic parameter of filters which is obtained from the diagram of filtration efficiency (if filter with fineness code $k$ is selected as a suction filter then $c_{1}=c_{1 k}$; if filter with fineness code $l$ is selected as a pressure filter then $c_{2}=c_{2 l}$; if filter with fineness code $u$ is selected as a suction filter then $c_{3}=c_{3 u}$ )

$C_{1 k}$ : Contaminant-holding capacity of suction filter with fineness code $k(\mathrm{~g})$

$C_{2 l}$ : $\quad$ Contaminant-holding capacity of pressure filter with fineness code $l(\mathrm{~g})$

$C_{3 u}$ : Contaminant-holding capacity of return filter with fineness code $u(\mathrm{~g})$

$\mathrm{C}_{4}$ : $\quad$ Contaminant-holding capacity of bypass filter (g)

$C_{k}^{(q i)}: \quad$ Cumulative function of the contaminant-holding capacity of filter $k(\mathrm{~g})$ with the probability $q_{i}$ of violating constraint $i$ and $k=1,2,3,4$

$C_{k}$ : Contaminant-holding capacity of filter $k(\mathrm{~g}$, $k=1,2,3,4)$

$D_{m}: \quad$ Average diameter of particle diameter range $m$ $(\mu \mathrm{m})$

$\mathrm{ER}_{k}: \quad$ Expenditure for replacement of filter element $k$ (RMB ¥)

$F_{\text {cost }}: \quad$ Total cost of the filtration system (RMB $\left.¥\right)$

$F_{\text {cost-opt }}$ : Optimal objective function values of the total cost of the filtration system (RMB $¥$ )

$F_{k}: \quad$ Binary variable, if filter $k$ exists then $F_{k}=1$; otherwise $F_{k}=0 ; k=1,2,3,4$

$\mathrm{LM}_{k}$ : Economic loss due to downtime caused by maintenance of filter element $k$ (RMB $¥)$

$m$ : $\quad$ Sequential number of a given particle diameter range, $m=1,2, \ldots, M$

$M$ : Maximum sequential number of a given particle diameter range

$n$ : $\quad$ Working period number of filter, $n=1,2, \ldots, N$

$N$ : $\quad$ Maximum period number during a planning horizon

$N F_{\text {inm }}$ : Contamination level in segment $i$ and period $n$ for a given particle diameter range $m(i=0$ for the suction line, $i=1$ for the pump inlet, $i=2$ for the inlet of the complex component, and $i=3$ for the return line) (number of particles/mL)

$N F_{0 n m}^{ \pm}$: An initial contamination level in segment 0 and period $n$ for a given particle diameter range $m$

$R_{k x_{i}}$ : Contaminant ingression and generation rates of contaminant particle diameter larger than $x_{i}$ in segment $k$ (number of particles/min; $k=1$ for the pump, $k=2$ for the complex component, and $k=3$ for the oil reservoir)

$\operatorname{Pr}(x)$ : Probability function

$p_{i}$ : $\quad$ Probability $p_{i}$ of violating constraint $i$
Q: $\quad$ Flow rate through the main circuit $(\mathrm{mL} / \mathrm{min})$

$Q_{b}: \quad$ Flow rate of the bypass system $(\mathrm{mL} / \mathrm{min})$

$R_{\text {jnm }}$ : Contaminant ingression/generation rate of component $j$ in period $n$ for a given particle diameter range $m$ (number of particles/min)

$S_{j n m}^{(q i)}$ : Cumulative function of contaminant tolerance level of component $j$ in period $n$ for a given particle diameter range $m$ ( $j=1$ for the pump, $j=2$ for control components and actuators) with the probability $q_{i}$ of violating constraint $i$

$S_{j n m}$ : Contaminant tolerance level of component $j(j=1,2)$ in period $n$ for a given particle diameter range $m$ (number of particles $/ \mathrm{mL}$ )

$T: \quad$ Circulation time in which the fluid flows through the whole hydraulic system ( $\mathrm{min}$ )

$V: \quad$ Fluid volume in the oil reservoir $(\mathrm{mL})$

$\mathrm{WM}_{k}$ : Worker wage spent on maintaining filter $k$ (RMB $¥)$ and/or constraint degree of satisfaction for the fuzzy objective and/or constraint.

\section{Conflict of Interests}

The authors declare that there is no conflict of interests regarding the publication of this paper.

\section{Acknowledgments}

The authors would like to thank the Natural Science Foundations of China (nos. 51075007 and 51375018), National High-Tech R\&D (863) Program (no. 2012AA091103), and The Importation and Development of High-Caliber Talents Project of Beijing Municipal Institutions (CIT\&TCD 20130316) for funding this research. The authors would like to thank the anonymous reviewers for their insightful comments and suggestions that were very helpful for improving the paper.

\section{References}

[1] R. H. Frith and W. Scott, "Control of solids contamination in hydraulic systems-an overview," Wear, vol. 165, no. 1, pp. 6974, 1993.

[2] C. D. David, "Keeping hydraulic fluid clean," Plant Engineering, vol. 51, no. 3, pp. 86-88, 1997.

[3] B. Poeth, "Proactive maintenance," Fluid Power Journal, vol. 12, no. 1, pp. 28-30, 2005.

[4] S. L. Nie, G. H. Huang, Y. Q. Zhu, Z. Y. Li, and Y. P. Li, "SEWHAPM: development of a water hydraulic axial piston motor for underwater tool systems," Journal of Mechanical Engineering Science, vol. 219, no. 7, pp. 639-656, 2005.

[5] S. L. Nie, G. H. Huang, and Y. P. Li, "Tribological study on hydrostatic slipper bearing with annular orifice damper for 
water hydraulic axial piston motor," Tribology International, vol. 39, no. 11, pp. 1342-1354, 2006.

[6] S. L. Nie, G. H. Huang, Y. P. Li, Y. S. Yang, and Y. Q. Zhu, "Research on low cavitation in water hydraulic two-stage throttle poppet valve," Journal of Process Mechanical Engineering, vol. 220, no. 3, pp. 167-179, 2006.

[7] C. Y. Yao and J. Y. Zhao, "Application of silting principle to online monitoring oil contamination in hydraulic system," China Mechanical Engineering, vol. 17, no. 2, pp. 112-124, 2006.

[8] S. L. Nie, Y. P. Li, X. Y. Shi, G. H. Huang, and B. Hu, "An IPINP model for the assessment of filter allocation and replacement strategies in a hydraulic contamination control system under uncertainty," Journal of Mechanical Engineering Science, vol. 223, no. 4, pp. 999-1015, 2009.

[9] S. L. Nie, Z. Hu, Y. P. Li, and G. H. Huang, "Non-linear programming for filter management in a fluid power system with uncertainty," Journal of Power and Energy, vol. 224, no. 2, pp. 185-201, 2010.

[10] S. L. Nie, Y. P. Li, Z. B. Xiong, G. H. Huang, and B. Hu, "IFQP: a hybrid optimization method for filter management in fluid power systems under uncertainty," Engineering Optimization, vol. 42, no. 1, pp. 45-68, 2010.

[11] S. L. Nie, Y. L. Zheng, Y. P. Li, S. Peng, and G. H. Huang, "IFCIP: an integrated optimization method for planning filters in fluid power systems under uncertainty," Engineering Optimization, vol. 43, no. 3, pp. 329-348, 2011.

[12] I. M. Stancu-Minasian, "Overview of different approaches for solving stochastic programming problems with multiple objective functions," in Stochastic Versus Fuzzy Approaches to Multiobjective Mathematical Programming under Uncertainty, pp. 71-101, Kluwer Academic, Dordrecht, The Netherlands, 1990.

[13] A. Charnes and W. W. Cooper, "Chance-constrained programming," Management Science, vol. 6, no. 1, pp. 73-79, 1959.

[14] A. Charnes and W. W. Cooper, "Response to 'decision problems under risk and chance constrained programming: dilemmas in the transitions,' Management Science, vol. 29, no. 6, pp. 750-753, 1983.

[15] D. P. Loucks, J. R. Stedinger, and D. A. Haith, Water Resource Systems Planning and Analysis, Prentice-Hall, Englewood Cliffs, NJ, USA, 1981.

[16] Y. P. Li, G. H. Huang, S. L. Nie, and X. S. Qin, "ITCLP: an inexact two-stage chance-constrained program for planning waste management systems," Resources, Conservation and Recycling, vol. 49, no. 3, pp. 284-307, 2007.

[17] Y. P. Li, G. H. Huang, S. L. Nie, and L. Liu, "Inexact multistage stochastic integer programming for water resources management under uncertainty," Journal of Environmental Management, vol. 88, no. 1, pp. 93-107, 2008.

[18] B. Liu, Theory and Practice of Uncertain Programming, Springer, Berlin, Germany, 2nd edition, 2009.

[19] J. H. Ellis, E. A. McBean, and G. J. Farquhar, "Chanceconstrained/stochastic linear programming model for acid rain abatement-I. Complete colinearity and noncolinearity," Atmospheric Environment, vol. 19, no. 6, pp. 925-937, 1985.

[20] J. H. Ellis, E. A. McBean, and G. J. Farquhar, "Chanceconstrained/stochastic linear programming model for acid rain abatement-II. Limited colinearity," Atmospheric Environment, vol. 20, no. 3, pp. 501-511, 1986.
[21] G. H. Huang, "A hybrid inexact-stochastic water management model," European Journal of Operational Research, vol. 107, no. 1, pp. 137-158, 1998.

[22] G. H. Huang, N. Sae-Lim, L. Liu, and Z. Chen, "An intervalparameter fuzzy-stochastic programming approach for municipal solid waste management and planning," Environmental Modeling \& Assessment, vol. 6, no. 4, pp. 271-283, 2001.

[23] L. Liu, G. H. Huang, Y. Liu, G. A. Fuller, and G. M. Zeng, "A fuzzy-stochastic robust programming model for regional air quality management under uncertainty," Engineering Optimization, vol. 35, no. 2, pp. 177-199, 2003.

[24] D. R. Morgan, J. W. Eheart, and A. J. Valocchi, "Aquifer remediation design under uncertainty using a new chance constrained programming technique," Water Resources Research, vol. 29, no. 3, pp. 551-568, 1993.

[25] X. Huang, "Chance-constrained programming models for capital budgeting with NPV as fuzzy parameters," Journal of Computational and Applied Mathematics, vol. 198, no. 1, pp. 149159, 2007.

[26] X. Huang, "Optimal project selection with random fuzzy parameters," International Journal of Production Economics, vol. 106, no. 2, pp. 513-522, 2007.

[27] M. Roubens and J. Teghem Jr., "Comparison of methodologies for fuzzy and stochastic multi-objective programming," Fuzzy Sets and Systems, vol. 42, no. 1, pp. 119-132, 1991.

[28] T. Watanabe and H. Ellis, "A joint chance-constrained programming model with row dependence," European Journal of Operational Research, vol. 77, no. 2, pp. 325-343, 1994.

[29] Y. Zare and A. Daneshmand, "A linear approximation method for solving a special class of the chance constrained programming problem," European Journal of Operational Research, vol. 80, no. 1, pp. 213-225, 1995.

[30] G. Infanger and D. P. Morton, "Cut sharing for multistage stochastic linear programs with interstage dependency," Mathematical Programming B, vol. 75, no. 2, pp. 241-251, 1996.

[31] Y. R. Fan and G. H. Huang, "A robust two-step method for solving interval linear programming problems within an environmental management context," Journal of Environmental Informatics, vol. 19, no. 1, pp. 1-12, 2012.

[32] G. H. Huang, B. W. Baetz, and G. G. Patry, "A grey fuzzy linear programming approach for municipal solid waste management planning under uncertainty," Civil Engineering Systems, vol. 10, no. 2, pp. 123-146, 1993.

[33] Z. X. Xia, Contamination Control of Hydraulic System, China Machine Press, Beijing, China, 1992, (Chinese).

[34] G. H. Huang, B. W. Baetz, and G. G. Patry, "Grey integer programming: an application to waste management planning under uncertainty," European Journal of Operational Research, vol. 83, no. 3, pp. 594-620, 1995.

[35] D. Norvelle, "Cleaning up clutter in fluid analysis," Machine Design, vol. 69, no. 11, pp. 48-52, 1997.

[36] N. C. P. Edirisinghe, E. I. Patterson, and N. Saadouli, “Capacity planning model for a multipurpose water reservoir with targetpriority operation," Annals of Operations Research, vol. 100, no. 1-4, pp. 273-303, 2000.

[37] Y. P. Li, G. H. Huang, S. L. Nie, and Y. F. Huang, "IFTSIP: interval fuzzy two-stage stochastic mixed-integer linear programming: a case study for environmental management and planning," Civil 
Engineering and Environmental Systems, vol. 23, no. 2, pp. 73-99, 2006.

[38] J. R. Birge and F. V. Louveaux, "A multicut algorithm for two-stage stochastic linear programs," European Journal of Operational Research, vol. 34, no. 3, pp. 384-392, 1988.

[39] S. Peng, Research on application of two-stage stochastic, chanceconstrained and fuzzy-parameter optimization in contamination control for hydraulic systems [Ph.D.dissertation], University of Science and Technology, Huazhong, China, 2009, (Chinese). 


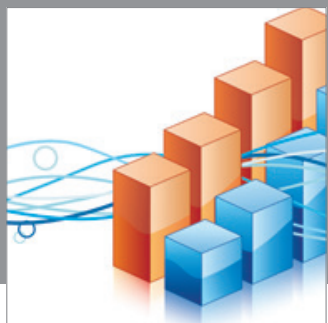

Advances in

Operations Research

mansans

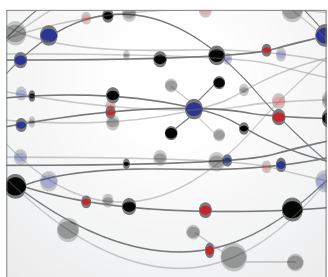

The Scientific World Journal
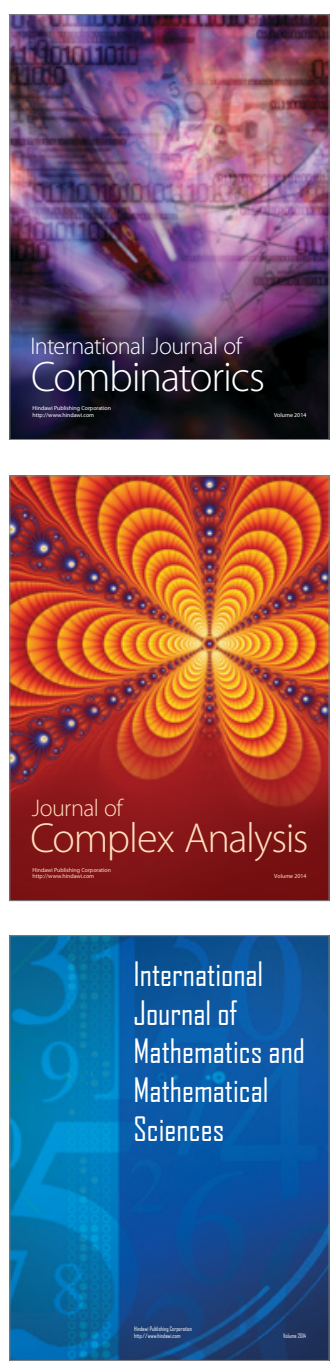
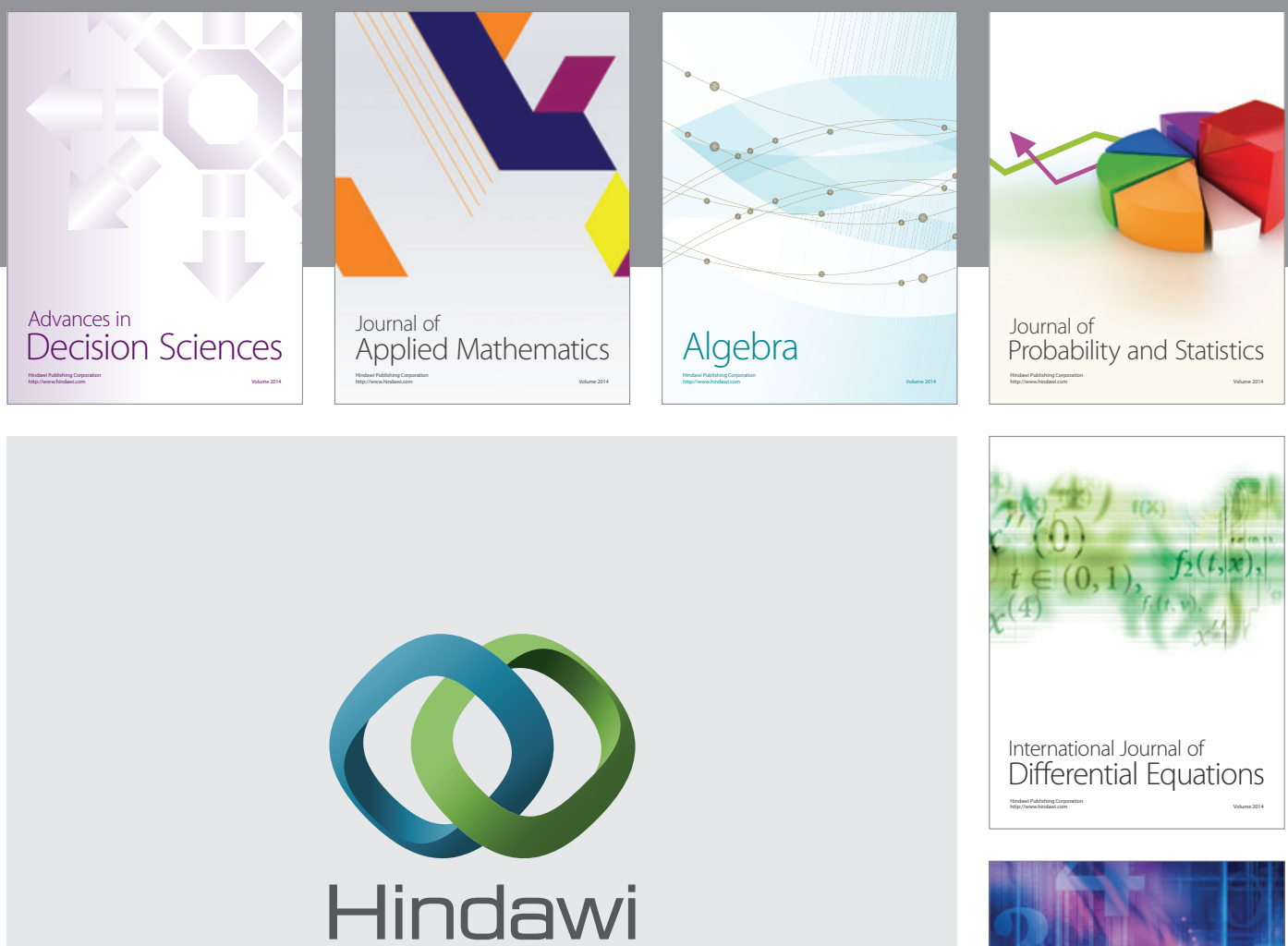

Submit your manuscripts at http://www.hindawi.com
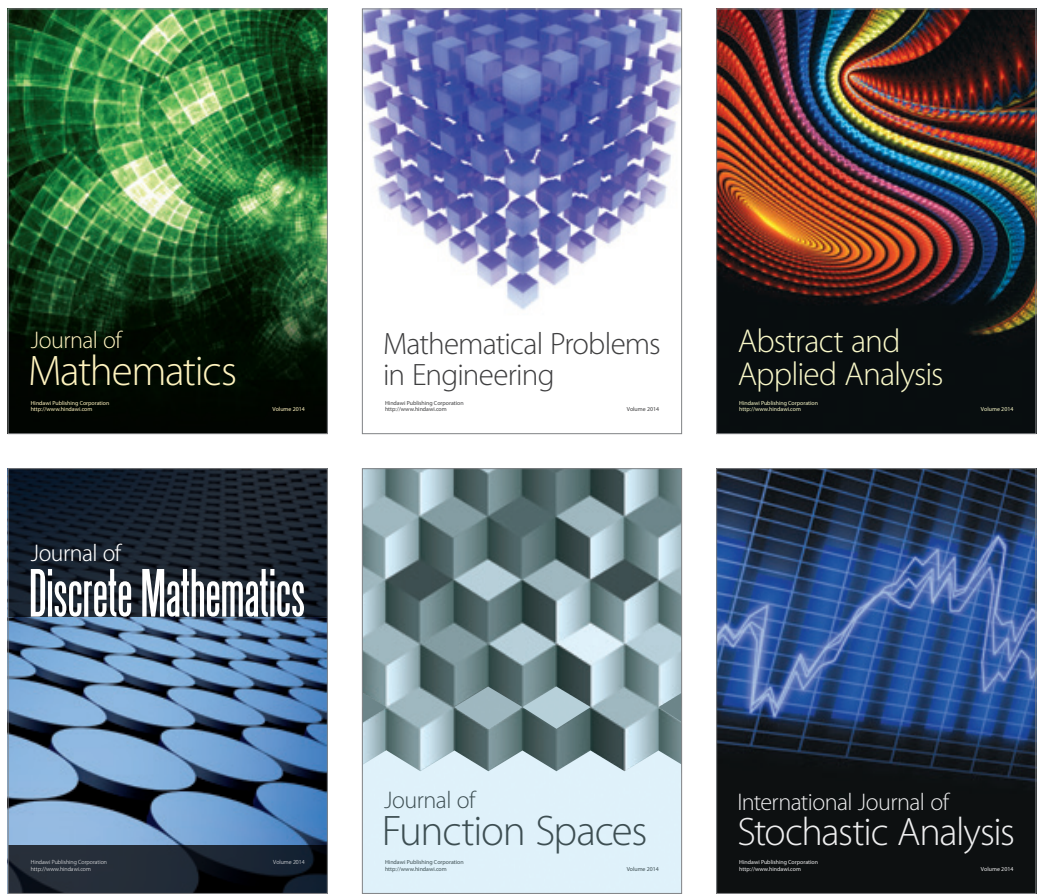

Journal of

Function Spaces

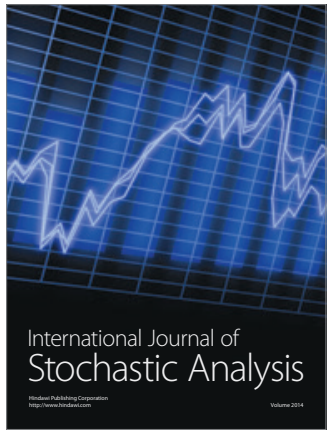

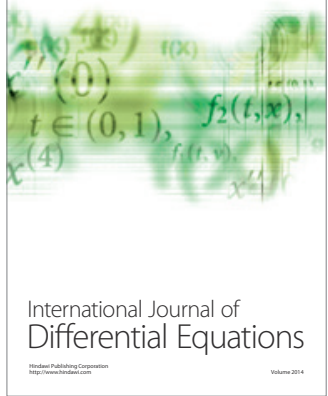
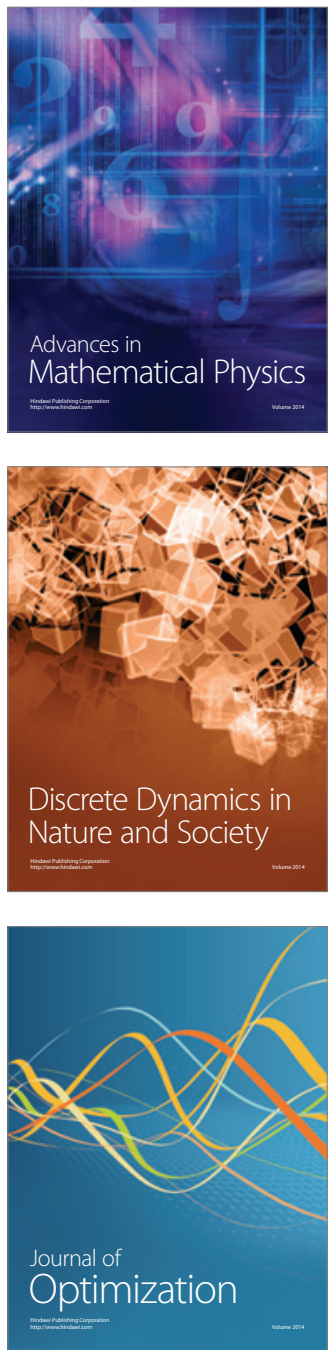